\title{
Candidíase e candidemia neonatal: Revisão de literatura
}

\author{
Neonatal candidiasis and candidemia: A literature review \\ Candidiasis y candidemia neonatales: Revisión de la literatura
}

Recebido: 26/03/2021 | Revisado: 08/04/2021 | Aceito: 11/04/2021 | Publicado: 22/04/2021

\author{
Gabriela Alves Luz \\ ORCID: https://orcid.org/0000-0002-6875-9561 \\ Universidade Ceuma, Brasil \\ E-mail: alvesgabriela2001@gmail.com \\ Gabriela Coleta Schneider \\ ORCID: https://orcid.org/0000-0002-4309-3767 \\ Universidade Ceuma, Brasi \\ E-mail: gabrielacoleta@hotmail.com \\ Monique Santos do Carmo \\ ORCID: https://orcid.org/0000-0003-0364-1420 \\ Universidade Ceuma, Brasil \\ E-mail: carmo.monique@outlook.com
}

\begin{abstract}
Resumo
O objetivo desse estudo foi realizar uma revisão de literatura sobre a candidíase e candidemia neonatal abordando a etiopatogenia, epidemiologia, fatores de virulência, fatores de risco, manifestações clínicas, diagnóstico, tratamento, prevenção, resposta imunológica, padrão de susceptibilidade aos antifúngicos, estratégias para redução da morbimortalidade e perspectivas futuras. Foram elencados artigos publicados nos últimos dez anos nas bases de dados PubMed, Medline e Google Scholar. A candidíase e a candidemia neonatal são importantes causas de morbidade e mortalidade em todo o mundo, de forma que mais de 750.000 casos são registrados anualmente. Os recém-nascidos mais susceptíveis são os prematuros, de baixo peso, imunosuprimidos, sob o uso de cateter, ventilação mecânica e nutrição parenteral. É necessário um controle mais rígido dos fatores de risco dentro do ambiente hospitalar associado ao cuidado dos profissionais, além do uso racional de antifúngicos seja na prevenção ou tratamento da candidíase e candidemia. É importante ressaltar também sobre a mudança gradual no curso epidemiológico dessas patologias pela emergência de novas espécies não albicans que podem representar sério risco para os neonatos. Políticas públicas direcionadas para o manejo adequado da candidíase e candidemia são necessárias.
\end{abstract}

Palavras-chave: Candidíase; Candidemia; Candida; Neonatos.

\begin{abstract}
The aim of this study was to conduct a literature review on neonatal candidiasis and candidemia, addressing etiopathogenesis, epidemiology, virulence factors, risk factors, clinical manifestations, diagnosis, treatment, prevention, immune response, susceptibility to antifungals, strategies for reduction of morbidity and mortality and future perspectives. Articles published in the last ten years in the PubMed, Medline and Google Scholar databases were listed. Candidiasis and neonatal candidemia are important causes of morbidity and mortality worldwide, so that more than 750,000 cases are registered annually. The most susceptible newborns are premature, low birth weight, immunosuppressed, using catheters, mechanical ventilation and parenteral nutrition. Stricter control of risk factors within the hospital environment associated with the care of professionals is required, in addition to the rational use of antifungals in the prevention or treatment of candidiasis and candidemia. It is also important to highlight the gradual change in the epidemiological course of these pathologies due to the emergence of new non-albicans species that can represent a serious risk for neonates. Public policies aimed at the proper management of candidiasis and candidemia are necessary.
\end{abstract}

Keywords: Candidiasis; Candidemia; Candida; Neonates.

\section{Resumen}

El objetivo de este estudio fue realizar una revisión de la literatura sobre candidiasis y candidemia neonatal, abordando etiopatogenia, epidemiología, factores de virulencia, factores de riesgo, manifestaciones clínicas, diagnóstico, tratamiento, prevención, respuesta inmunológica, susceptibilidad a antifúngicos, estrategias de reducción de la morbilidad y mortalidad y perspectivas de futuro. Se enumeraron los artículos publicados en los últimos diez años en las bases de datos PubMed, Medline y Google Scholar. La candidiasis y la candidemia neonatal son causas importantes de morbilidad y mortalidad a nivel mundial, por lo que se registran más de 750.000 casos al año. Los recién nacidos más susceptibles son los prematuros, de bajo peso al nacer, inmunosuprimidos, que utilizan catéteres, ventilación mecánica y nutrición parenteral. Se requiere un control más estricto de los factores de riesgo dentro del 
ámbito hospitalario asociados a la atención de los profesionales, además del uso racional de antifúngicos en la prevención o tratamiento de la candidiasis y candidemia. También es importante destacar el cambio paulatino en el curso epidemiológico de estas patologías debido a la aparición de nuevas especies no albicans que pueden representar un grave riesgo para los neonatos. Son necesarias políticas públicas orientadas al manejo adecuado de la candidiasis y la candidemia.

Palabras clave: Candidiasis; Candidemia;Candida; Recién nacidos.

\section{Introdução}

A candidíase invasiva e candidemia $(\mathrm{CI} / \mathrm{C})$ são condições clínicas de grande repercussão em neonatos. Cerca de 2 a $20 \%$ dos recém-nascidos (RN) prematuros são acometidos, sendo que 10\% dos casos de sepse ocorre em RN com baixo peso $(<1.000 \mathrm{~g})$ (Benjamin et al., 2010; Fu et al., 2018). Apesar da progressão na assistência neonatal no último decênio, o índice de mortalidade da CI/C varia de 36-63\% entre diferentes países (Pfaller et al., 2012; Tragiannidis et al., 2012; Montagna et al., 2014; Vasileiou, Apsemidou, Vyzantiadis, \& Tragiannidis, 2018).

O principal agente etiológico da CI/C é a levedura Candida albicans, que representa cerca de $60 \%$ das espécies de Candida isoladas em amostras clínicas (Calley \& Warris, 2017). Entretanto, a epidemiologia da infecção vem sofrendo alterações com o aumento de casos relacionados a outras espécies do gênero, tais como C.tropicalis, C.parapsilosis, C.glabrata e C.krusei (Mantadakis \& Tragiannidis, 2019).

O fungo faz parte da microbiota da pele e do trato gastrointestinal do neonato, entretanto, pode ser translocado de sítio à partir da realização de procedimentos médicos, da manipulação pela equipe multiprofissional, da exposição à superfícies contaminadas e de modo vertical (Arsenault \& Bliss, 2015). Depois de ter acesso às mucosas e corrente sanguínea, Candida spp. apresenta maior afinidade pela invasão do sistema nervoso central, rins, fígado, baço, coração e retina, gerando complicações que comprometem suas funções (Adams-Chapman et al., 2013; Wang, Dai, Liu, \& Li, 2013; Auriti et al., 2016; Mantadakis \& Tragiannidis, 2019).

Do ponto de vista clínico, a candidíase neonatal pode apresentar-se sob as formas de candidíase orofaríngea (incluindo aftas, glossite, estomatite), cutânea (incluindo intertrigo - frequentemente observada na axila, virilha, pregas glúteas, espaços interdigitais e umbigo), mucocutânea (se estende ao longo da pele, mucosas e unhas) e sistêmica, com difusão para vários orgãos vitais do corpo (Dutta, 2019).

Por outro lado, a candidemia consiste na presença das leveduras de Candida spp. na via hematogênica. É a principal causa de infecções fúngicas invasivas em crianças hospitalizadas e o terceiro tipo de infecção nosocomial da corrente sanguínea mais comum em todo o mundo, precedida pelas bacteremias por Staphylococcus coagulase-negativo, S. aureus e Enterococcus spp (Mantadakis, Pana \& Zaoutis, 2018).

Nas Unidades Pediátricas de Cuidados Intensivos, os neonatos mais susceptiveis à CI/C são aqueles submetidos a procedimentos invasivos como inserção de cateter vascular central, intubação endotraqueal, ventilação mecânica, nutrição parenteral, portadores de doenças gastrointestinais, crônicas, câncer e de ampla exposição a antibioticoterapias (Mantadakis et al., 2018). Um estudo prévio constatou que dos neonatos que sobrevivem à candidemia, cerca de $58 \%$ frequentemente apresentam comprometimento no desenvolvimento neurológico (Benjamin et al., 2006).

O padrão-ouro para diagnóstico da CI/C são as hemoculturas, mas, o painel T2Candida é uma técnica mais recente e sensível que permite uma ampla detecção espécie-específica das leveduras desse gênero (Hamula et al., 2016). Apesar da existência de técnicas eficazes de identificação microbiológica, a falta de especificidade nos sinais e sintomas retarda o diagnóstico e aumenta o risco de mortalidade (Chang et al., 2013). Logo, o diagnóstico precoce com intervenção imediata é fundamental para o controle da infecção e redução da morbimortalidade.

Diante desse contexto, o objetivo desse estudo foi realizar uma revisão de literatura sobre a candidíase e candidemia neonatal abordando a etiopatogenia, epidemiologia, fatores de virulência, fatores de risco, manifestações clínicas, diagnóstico, 
tratamento, prevenção, resposta imunológica, padrão de susceptibilidade aos antifúngicos, estratégias para redução da morbimortalidade e perspectivas futuras para o tratamento dessas patologias.

\section{Metodologia}

O estudo foi desenvolvido por revisão do tipo sistemática à partir da seleção de artigos publicados em inglês e português nos últimos dez anos dentro dos bancos de dados PubMed, Medline e Google Scholar, utilizando-se os seguintes descritores: candidíase, candidemia, neonatal, etiopatogenia, epidemiologia, fatores de virulência, fatores de risco, manifestações clínicas, diagnóstico, tratamento, prevenção, resposta imunológica, padrão de susceptibilidade aos antifúngicos e estratégias para redução da morbimortalidade, de forma combinada. Os critérios de exclusão foram artigos que se repetiam nas bases de dados e que fugiam à temática (Estrela, 2018).

\section{Resultados e Discussão}

\subsection{Candidíase Neonatal}

\subsubsection{Etiopatogenia}

A candidíase neonatal é definida como uma infecção aguda ou crônica da pele, das membranas mucosas e, mais raramente, dos órgãos internos causada por várias espécies do gênero Candida, tais como C.glabrata, C.tropicalis, C.parapsilosis, C.krusei, sendo C.albicans a causa mais frequente na grande maioria dos casos. Recém-nascidos e lactentes são fisiologicamente mais suscetíveis à infecção por Candida, podendo manifestar-se como candidíase relacionada a fraldas, candidíase oral ou intertrigos (Kliegman, Geme, Blum, Shah, \& Tasker, 2020).

A colonização está associada ao tipo de parto. A infecção ocorre quando há um desequilíbrio na relação parasitahospedeiro, resultante da disbiose da microbiota normal e ruptura das defesas inatas. Sua patogênese é iniciada quando as leveduras aderem aos resíduos de açúcar na mucosa bucal ou às proteínas da matriz extracelular das células epiteliais. Além disso, quando há dano ao epitélio e a integridade das barreiras da mucosa é comprometida, uma maior quantidade de proteínas da matriz extracelular são expostas, aumentando a adesão e disseminação do microrganismo (Arsenault \& Bliss, 2015).

Cerca de 60\% dos profissionais de saúde têm Candida spp. na microbiota da pele, na qual C.parapsilosis é a mais frequente, podendo ser facilmente veiculada aos neonatos e facilitar o risco de infecção (Brühwasser et al., 2016).

\subsubsection{Epidemiologia}

A incidência das infecções invasivas por Candida em neonatos é de aproximadamente 5 a 10 casos por 100.000 bebês nascidos vivos (Benedict et al., 2018; Blyth et al., 2009; Lausch et al., 2019). Sua epidemiologia em UTIN varia de acordo com a localização geográfica. Um estudo realizado nos Estados Unidos que avaliou a ocorrência de infecções fúngicas invasivas em UTIN (Unidade de Terapia Intensiva Neonatal), encontrou incidência de 9,8/1000 pacientes admitidos por ano na unidade. Além disso, foi constatada prevalência de Candida spp., totalizando 95,2\% das infecções (Agarwal, Agarwal, Chen, Lua, \& Ang, 2017). Uma pesquisa desenvolvida pelo sistema de vigilância do Reino Unido em 30 UTIN entre 2005 a 2014, identificou espécies de Candida como o patógeno causador de $4 \%$ de todos os casos de sepse neonatal de início tardio (definida como $\geq 48$ horas após o nascimento) em culturas positivas (Cailes et al., 2018).

Alguns autores tem relatado uma mudança gradual de infecção de Candida albicans para espécies não- albicans. As taxas de mortalidade permanecem altas, dependendo do patógeno fúngico isolado e da condição subjacente do paciente pediátrico (Pana, Roilides, Warris, Groll, \& Zaoutis, 2017). Um estudo retrospectivo realizado no Hospital universitário terciário no sudoeste da China constatou um total de 86 episódios de candidíase invasiva (CI). A incidência média anual de CI 
foi de 1,04 a cada 1000 admissões, sendo 2.72 / 1000 admissões neonatais e 0.51 / 1000 admissões não neonatais (dentre elas 0,77 / 1000 admissões infantis e 0,41/ 1000 internações pediátricas). As taxas médias anuais de incidência de C.albicans foram 0,51 / 1000 admissões e infecções não-albicans por Candida foram 0,53 / 1000 admissões (Zeng et al., 2020).

Houve um aumento da incidência de CI entre todos os pacientes pediátricos internados de 0,49 episódios / 1000 admissões em 2013 para 1,78 episódios / 1000 admissões em 2016, porém, ocorreu uma diminuição de 1,79 episódios / 1000 admissões em 2017 para 0,87 episódios / 1000 admissões em 2019, enquanto a incidência de CI entre pacientes neonatais internados apresentou um aumento de 0,42 episódios / 1000 admissões em 2013 para 5,71 episódios / 1000 admissões em 2016, mas diminuiu de 5,04 / 1000 admissões em 2017 para 1,83 / 1000 admissões em 2019. Dentre as taxas de incidência (episódios / 1000 admissões) de C.albicans foi observado a taxa de 0,39 em 2013 para 1,07 em 2016 e de 1,07 em 2016 para 0,39 em 2019 e Candida não-albicans de 0,10 em 2013 para 1,06 em 2017 e de 1,06 em 2017 para 0,48 em 2019 (Zeng et al., 2020).

Dentre as espécies mais prevalentes nesse estudo, C.albicans foi predominante $(66,7 \%)$ na Unidade de Terapia Intensiva Pediátrica (UTIP), enquanto a C.glabrata foi a espécie predominante $(55,0 \%)$ entre as enfermarias pediátricas gerais. Na UTIN, C.albicans e C.glabrata eram ambas espécies dominantes, respondendo pela mesma proporção (48,1\%). Dentre todos os pacientes internados, neonatos e não neonatos (incluindo bebês e crianças), C.albicans foi a espécie predominante em pacientes recém-nascidos e não neonatos (48,1\% e 50,0\%, respectivamente) (Zeng et al., 2020).

\subsubsection{Fatores de virulência}

Os fatores de virulência conferem vantagens seletivas para que os micro-organismos consigam se instalar nos neonatos e causar a infecção, e dentre eles destacam-se a secreção de hemolisinas, proteases, fosfolipases, DNAse e formação de biofilmes (Seneviratne et al., 2016; Mandelblat et al., 2017). A intensidade e expressão destes fatores variam conforme a espécie, o sítio do hospedeiro (mucosas, pele), tipo e gravidade do processo infeccioso, bem como o estado imunológico do indivíduo (Lahkar, Saikia, Patgiri, Nath, \& Das, 2017)

As hemolisinas permitem que a levedura obtenha o ferro através da destruição das hemácias, facilita a invasão das hifas e consequentemente a manutenção e disseminação do processo infeccioso (França, Furlaneto-Maia, \& Furlaneto, 2017). As proteases, fosfolipases e DNAse são utilizadas para lise das proteínas, fosfolipídeos e DNA das células do hospedeiro, respectivamente (de Melo Riceto et al., 2015; de Paula Menezes et al., 2019).

Outro fator importante é a secreção de biofilmes, estrutura complexa composta por leveduras, hifas e pseudo-hifas que são envoltas por uma matriz extracelular que permite a colonização e fixação do microrganismo aos tecidos e superfícies de dispositivos médicos invasivos (de Paula Menezes et al., 2019). Os biofilmes também são importantes estruturas de resistência à ação de antifúngicos e protegem as leveduras da resposta imune do hospedeiro. A capacidade de formar biofilme é espécieespecífica, porém, é mais comum em C.tropicalis, seguida de C.albicans, C.glabrata, C.parapsilosis e C.dubliniensis (Deorukhkar \& Saini, 2015).

\subsubsection{Fatores de risco}

Os principais fatores de risco associados a infecções neonatais incluem o baixo peso ao nascer $(<1500 \mathrm{~g})$ e a pouca idade gestacional (< 28 semanas), sendo condições predominantes para infecções invasivas, em especial as desencadeadas por espécies de Candida, denominadas candidíases invasivas. Isso ocorre por causa da imaturidade do sistema imunológico do recém-nascido (RN) associada a não integridade da pele e mucosas (Arsenault \& Bliss, 2015; Cantey \& Milstone, 2015; Boo \& Cheah, 2016; Calley \& Warris, 2017).

Os RN extremamente prematuros constantemente apresentam complicações em decorrência da sua condição, sendo as 
mais prevalentes a displasia broncopulmonar e enterocolite necrotizante, sendo diretamente relacionadas à ocorrência de sepse. Alguns estudos mostraram que a taxa de sepse tardia foi de $46 \%$ em neonatos nascidos com 25 semanas de gestação, $28 \% \mathrm{em}$ RN entre 25 e 28 semanas e aproximadamente $10 \%$ em RN nascidos com 29 a 32 semanas (Cantey \& Milstone, 2015).

Outros fatores de risco adicionais incluem o uso de bloqueadores de receptores de $\mathrm{H} 2$, que alteram o $\mathrm{pH}$ gástrico e a microbiota do indivíduo, facilitando a reprodução de Candida spp. Além disso, o uso da nutrição parenteral, em especial de infusões lipídicas, propicia um ambiente lipofílico, ideal para a proliferação de espécies de Candida. Todos estes fatores citados, exceto aqueles relacionados à prematuridade, podem ser modificáveis, com sua utilização consciente e limitada para a redução da ocorrência de candidíase invasiva (Arsenault \& Bliss, 2015).

Em um estudo de coorte de 12 anos, foi observado que a maioria dos casos de candidíase invasiva neonatal ocorreu em recém-nascidos de muito baixo peso (<1500 gramas) e a idade gestacional média nesse grupo foi de 27,8 semanas. Dentre os fatores predisponentes mais comuns estão o uso de cateter intravenoso central (CVC) (94,2\%), uso de antibióticos de amplo espectro $(91,8 \%)$, permanência na UTI $(69,3 \%)$, uso de nutrição parenteral $(64,6 \%)$ e subjacentes sequelas neurológicas $(36,0 \%)$ (Hsu et al., 2018).

No entanto, as doenças subjacentes e os principais fatores de risco foram diferentes entre a candidíase invasiva neonatal e os episódios pediátricos não neonatais. Enquanto os neonatos com candidíase invasiva eram mais propensos a receber nutrição parenteral total e ter doença pulmonar crônica, a candidemia pediátrica não neonatal tinha maior probabilidade de ocorrer em crianças com sequelas neurológicas subjacentes, dispositivos artificiais diferentes de CVC, neutropenia e agentes imunossupressores tratados com câncer (Hsu et al., 2018).

\subsubsection{Manifestações clínicas}

As manifestações clínicas podem ser do tipo mucucotânea, cutânea e sistêmica. As mucocutâneas acometem a cavidade oral com placas de exsudato esbranquiçado na mucosa, podendo ocorrer também glossite e queilite angular. Nesse tipo de apresentação clínica é possível também verificar alterações no canal vaginal, seja na forma de vulvovaginite ou balanopostite (Marostica, Villetti, Ferrelli, \& Barros, 2018).

A candidíase cutânea geralmente é resultante do uso contínuo de fraldas apertadas, manifestando-se pelo aparecimento de placas eritematosas, erosadas e com pústulas satélites principalmente em regiões de dobras (infra mamário, inguinal). A candidíase sistêmica é uma forma rara e grave de sepse nosocomial, sendo que seus principais fatores de risco são: prematuros extremos, peso de nascimento $<1.000 \mathrm{~g}$, hipoglicemia, uso de antibióticos de amplo espectro, uso prolongado de nutrição parenteral e presença de cateter central (Marostica et al., 2018).

Quando atingem o sistema nervoso central, as infecções causadas por Candida spp. frequentemente podem resultar em abscessos, porém, com parâmetros normais no líquido cefalorraquidiano (contagem de leucócitos, glicose e proteína). Observa-se também que a endoftalmite é complicação incomum, afetando <5\% das crianças com candidíase invasiva. O envolvimento renal geralmente complica a candidíase invasiva neonatal, podendo ser limitado à candidúria ou manifestar-se com infiltração difusa de Candida em todo o parênquima renal ou a presença de Candida e debris no sistema coletor. Outros órgãos afetados incluem os ossos, articulações, fígado e baço (Kliegman et al., 2020).

\subsubsection{Diagnóstico}

O diagnóstico de candidíase deve ser feito baseado na identificação dos grupos de risco mais frequentes da infecção. Dentre as opções diagnósticas disponíveis, as hemoculturas permitem a identificação das espécies e a possibilidade de estabelecer uma sensibilidade antifúngica, porém são limitadas por terem baixa sensibilidade diagnóstica. Novas estratégias estão sendo implementadas, que incluem técnicas moleculares que devem ser amplamente utilizadas para melhorar o 
diagnóstico, reduzir a mortalidade e diminuir o uso indevido de antifúngicos (Cortés, Ruiz, Melgarejo-Moreno, \& Lemos, 2020).

O diagnóstico é realizado através da utilização de técnicas que se complementam como a observação microscópica direta, cultura convencional, detecção de antígeno/anticorpos e amplificação de material genético pela reação em cadeia da polimerase (PCR) (Rocha, de Carvaho, \& Magalhães, 2019).

No diagnóstico molecular pode ser utilizado tanto os dependentes de hemocultura como os não dependentes. Nos dependentes, os testes são projetados para amostras de hemocultura positivas, sendo importante avaliar o tempo necessário para a positivação da hemocultura; como a carga do patógeno é alta em frascos de hemocultura positivos, a sensibilidade não é um desafio para esses ensaios. Em contrapartida, os diagnósticos moleculares independentes de hemocultura podem ser realizados diretamente em amostras de sangue total, soro ou plasma sem a necessidade de esperar por hemoculturas positivas. Essa segunda possibilidade apresenta maior potencial de economia em tempo maior quando comparada aos testes dependentes de hemocultura, mas como a carga de patógenos no sangue é baixa, a sensibilidade pode ser um problema (Camp, Spettel, \& Willinger, 2020).

Testes de amplo espectro também são utilizados, como exemplo os ensaios multiplex (panfúngicos) direcionados, em que o princípio é amplificar regiões-alvo conservadas que podem ser encontradas em todas as espécies de fungos. Os ensaios panfúngicos geralmente apresentam uma menor sensibilidade se comparado aos ensaios direcionados a certos patógenos com primers específicos da espécie, mas possuem uma capacidade de detectar todos os patógenos fúngicos (não apenas os mais frequentes). Um problema com o uso de PCR multiplex pode surgir do fato de que os médicos podem considerar que um resultado multiplex negativo é suficiente para descartar uma infecção fúngica invasiva (Camp et al., 2020).

Portanto, é importante especificar quais patógenos podem ser identificados nesses testes. A alta sensibilidade torna-os ferramentas atraentes para o diagnóstico precoce de CI. A PCR tem uma maior sensibilidade se comparada a hemocultura, mas mostra sua melhor eficácia quando usada em conjunto com hemoculturas e/ou testes adicionais. Além disso, o uso de técnicas moleculares para hemoculturas positivas permite uma identificação mais rápida de Candida spp (Camp et al., 2020).

Por isso, nos pacientes acometidos a intervenção medicamentosa deve ser precoce para minimizar os riscos de complicações e de resistência microbiana e, portanto, um diagnóstico laboratorial eficiente se faz necessário. O mais importante é estar atento ao RN, com atenção às formas de contágio, higiene correta das mãos, bem como saber e realizar higiene oral do RN de acordo com o protocolo de cuidados bucais na Unidade de Tratamento Intensivo (UTI) neonatal. Portanto, os profissionais devem conhecer quais os sinais clínicos e principalmente o que fazer para evitar o contagio (Rocha et al., 2019).

\subsubsection{Tratamento}

$\mathrm{Na}$ ausência de candidíase sistêmica, a terapia antifúngica tópica é o tratamento de escolha para manifestação cutânea congênita em crianças nascidas a termo. Caso ocorra a progressão da candidíase cutânea congênita para a sistêmica em prematuros, faz-se necessário o uso da terapia endovenosa (Kliegman et al., 2020).

No terapia cutânea, recomenda-se cremes como miconazol, cetoconazol ou isoconazol que podem ser utilizados por 2 a 4 semanas. Quando as mucosas são afetadas, recomenda-se o uso de nistatina suspensão oral por 7 a 14 dias. Nas formas mais extensas, em pacientes imunossuprimidos ou sem resposta ao tratamento tópico, recomenda-se o tratamento sistêmico com fluconazol ou anfotericina B desoxicolato (Marostica et al., 2018).

$\mathrm{Na}$ apresentação sistêmica faz-se necessário o tratamento por 21 dias a partir da última cultura positiva de Candida, sendo orientada com base nos testes de suscetibilidade. A anfotericina B desoxicolato é a base da terapia para candidíase sistêmica e é ativa contra as formas micelianas e de leveduras. O fluconazol também é utilizado com frequência para o 
tratamento de infecções invasivas neonatais, devido à baixa incidência de efeitos adversos e sua eficácia. As equinocandinas têm excelente atividade contra a maioria das espécies de Candida e são utilizadas com sucesso em pacientes com microrganismos resistentes ou em indivíduos que apresentaram falha terapêutica (Kliegman et al., 2020).

\subsubsection{Prevenção}

Atualmente, o uso da profilático do fluconazol está indicado apenas para menores de $1000 \mathrm{~g}$ de peso de nascimento e/ou prematuridade extrema (menores que 28 semanas de idade gestacional) em UTIN, pois apresentam elevadas taxas de incidência de CI (taxas maiores que 5\%). Em unidades com taxas menores que 5\%, a necessidade de profilaxia deve ser avaliada de acordo com fatores predisponentes para doença invasiva (Santolaya et al., 2013; Lollis, Bradshaw, \& Zukowsky, 2014; Pansieri, Pandolfini, Jacqz-Aigrain, Van Den Anker, \& Bonati, 2015).

Além da prevenção medicamentosa, é recomendada a implementação de medidas para controle dos fatores de risco, tais como: monitoramento da higienização das mãos dos profissionais de saúde, uso racional dos antibióticos de amplo espectro, cuidados com cateter venoso central e sua retirada precoce, redução do uso de bloqueadores $\mathrm{H} 2$ e do início precoce da dieta enteral (Hope et al., 2012; Pansieri et al., 2015).

\subsubsection{Resposta Imunológica}

Os fungos do gênero Candida podem estar presentes na microbiota dos indivíduos sadios, atuando como agentes comensais (Bachur, 2019). Quando ocorre acometimento nas barreiras mucosas ou funcionais da pele, desequilíbrios da microbiota ou defeitos nos mecanismos de defesa imunológica, há uma probabilidade acentuada de evolução para candidíase mucocutânea ou invasiva (Davidson, Netea, \& Kullberg, 2018).

A resposta imunológica rápida contra o patógeno, inicia-se pelo reconhecimento de padrões moleculares associados a patógenos conservados ("pathogen- associated molecular patterns" - PAMPs). Os PAMPs são distinguidos pelos receptores de reconhecimento de patógenos ("pathogen recognition receptors" - PRRs) (Netea, Joosten, Van Der Meer, Kullberg, \& Van De Veerdonk, 2015). A resposta imune se inicia após os receptores de fagócitos reconhecerem tais padrões moleculares. Entre estes, os principais são, receptores de manose; receptores semelhantes a Toll (“Toll-Like Receptors" - TLRs); receptores de varredura; receptor dectina-1 e e os receptores de lectina do tipo C ("C type Lectin Receptors" - CLRs) (De Bernardis, Graziani, Tirelli, \& Antonopoulou, 2018).

Os TLR2, TLR4 e TLR6 ficam ligados à membrana plasmática e reconhecem os constituintes da manoproteína da parede celular fúngica, de modo que o TLR2 e TLR4 são os principais envolvidos no reconhecimento de Candida spp. (Netea et al., 2015). A associação entre esses principais receptores com os PAMPs de Candida spp. induz a ativação da resposta imune inata, levando a síntese e liberação de quimiocinas proinflamatórias, citocinas e ativação do inflamossomo NLRP3, que é responsável pela conversão das inteleucinas IL-1beta e IL-18 para as formas ativas. Dessa forma, ocorre a ativação da inflamação e da resposta imune adaptativa. Simultaneamente, ocorrem fagocitose e morte do fungo, pelos neutrófilos (Davidson et al., 2018).

A resposta imunológica adaptativa ocorre após a fagocitose e processamento do fungo pelas células dendríticas que apresentam os antígenos gerados via moléculas de HLA (Antígeno Leucocitário Humano) classe II às células CD4 naive, resultando em uma resposta com padrão T-helper (Th) (Richardson \& Moyes, 2015). Estas células se proliferam na presença de citocinas inflamatórias e se diferenciam em Th1 e Th17. As células Th1 induzidas por IL-18, produzem interferon-gama (IFN- $\gamma$ ), um potencializador da atividade fungicida de neutrófilos e macrófagos. Já as células Th17 induzidas por IL-1beta, produzem IL-17 e IL-22, que promovem o recrutamento e ativação de neutrófilos, assim como a liberação de $\beta$-defensinas antifúngicas por células epiteliais (Netea et al., 2015). Seguida a fagocitose, os neutrófilos utilizam mecanismos oxidativos e 
não oxidativos para eliminar o fungo (Qin et al., 2016).

Ao nível das mucosas tem-se a via de sinalização da IL 17, importante no combate das infecções por fungos do gênero Candida. As células epiteliais e apresentadoras de antígeno, fazem o reconhecimento de Candida pelos receptores do tipo Lectina, que ativam o fator nuclear Kappa $\beta$ (NF-kB) com a participação do complexo proteico de sinalização CARD9/BCL10, resultando na produção de citocinas pró-inflamatórias como IL-1 $\beta$, IL-6 e TGF- $\alpha$. Estas induzem a diferenciação dos Linfócitos Th0 em linfócitos Th17 com a participação do fator de transcrição STAT 3. Esses linfócitos produzem IL-17 e IL23, recrutando neutrófilos com grande poder microbicida para as mucosas, os quais irão determinar a produção de defensinas e outros peptídeos com atividade microbiana, também secretados ao nível das mucosas. Quando ocorrem mutações com diversos padrões de herança genética, tem-se uma menor produção de IL-17 ou alterações nas vias de sinalização mencionadas acima, levando à candidíase mucocutânea crônica (CMC) (X. Wang, van de Veerdonk, \& Netea, 2016).

\subsubsection{Padrão de suscetibilidade às drogas antifúngicas}

O tratamento para as infecções fúngicas sistêmicas estão cada vez mais difíceis, devido à crescente resistência aos agentes antifúngicos entre as espécies de Candida, principalmente às equinocandinas (Sasso et al., 2017). C.glabrata tem uma suscetibilidade inferior ao fluconazol e pode desenvolver resistência cruzada a outros azólicos (Papadimitriou-Olivgeris et al., 2019). Portanto, é de suma importância a realização do teste de susceptibilidade aos antifúngicos para um correto manejo dos pacientes com candidíase, otimização do tratamento e redução das taxas de mortalidade e morbidade (Canassa \& da Cruz, 2019).

Um estudo que avaliou o perfil de sensibilidade das espécies de Candida encontradas em um hospital público no Mato Grosso do Sul, observou que a maioria dos isolados foi sensível in vitro aos antifúngicos testados. Foi encontrada uma cepa de C.parapsilosis resistente a anfotericina B. Entre os azóis (voriconazol e fluconazol), houve uma maior frequência de casos de diminuição da suscetibilidade, sendo observadas nas espécies C.albicans, C.parapsilosis e C.tropicalis. Uma cepa de Candida spp foi resistente à caspofungina e outra cepa de C.parapsilosis apresentou resistência a fluocitosina. Dentre as espécies, todas foram sensíveis à micafungina, sendo o fármaco de primeira escolha para tratamento nessa instituição (Canassa \& da Cruz, 2019).

Por outro lado, um estudo realizado em um hospital em Singapura constatou 99,2\% de suscetibilidade para anidulafungina, 98.9\% para a micafungina, 98,1\% para a caspofungina, 86,9\% para o voriconazol e 59,5\% para o fluconazol. C.albicans e C.parapsilosis mostraram alta suscetibilidade para o voriconazol e fluconazol, $>94 \%$. A resistência a equinocandina foi rara, ocorrendo em três isolados (C.glabrata, C.tropicalis e C.albicans) (Teo et al., 2017).

\subsubsection{Estratégias para redução da morbimortalidade}

A incidência da candidíase neonatal vem sofrendo um declínio nos últimos anos, sendo uma das causas mais importantes de morbidade e mortalidade entre bebês prematuros. O melhor entendimento da sua epidemiologia e de seus fatores de risco levou a modificações na abordagem desses pacientes, mas não eliminaram o risco dessas infecções. $O$ desenvolvimento de estudos com modelos animais adaptados ao hospedeiro neonatal tem fornecido insights sobre a virulência do patógeno e sua suscetibilidade ao hospedeiro. Com base nesses estudos é possível lançar mão de intervenções adaptadas a interação patógeno-hospedeiro, que são capazes de permitir estratégias preventivas e terapêuticas mais aprimoradas e direcionadas, reduzindo assim sua morbimortalidade (Arsenault \& Bliss, 2015).

Um estudo randomizado realizado em neonatos prematuros com baixo peso ao nascer $<1000 \mathrm{~g}$ que avaliou a resposta ao fluconazol e placebo, observou que houve a redução da colonização por Candida e as taxas de infecção fúngica invasiva sem surgimento de resistência aos medicamentos ou efeitos adversos durante longos períodos de tempo, sendo concluído que a 
profilaxia com fluconazol obteve o potencial de reduzir a mortalidade desses pacientes (Leibovitz, 2012).

A alta mortalidade dos neonatos pode ser atribuída às limitações dos testes de diagnóstico utilizados rotineiramente, seja pela quantidade insuficiente de testes e/ou demora na liberação do resultado (Greenberg \& Benjamin Jr, 2014; Kelly, Benjamin, \& Smith, 2015; Martin, Fanaroff, \& Walsh, 2016). Assim, uma das estratégias a ser adotadas para a redução da morbimortalidade nesses pacientes, seria a utilização de uma quantidade maior de testes e um resultado mais ágil tanto para se ter um diagnóstico mais abrangente, como para o tratamento precoce.

É necessário a realização de uma vigilância epidemiológica contínua nas unidades hospitalares de risco, permitindo o conhecimento das infecções mais frequentes e os micro-organismos mais comuns, tendo a possibilidade de adotar medidas para reduzir a ocorrência de infecção neonatal, refletindo na diminuição das taxas de morbimortalidade (Menezes, 2018).

\subsubsection{Perspectivas futuras: o que há de novo para o tratamento da candidíase?}

Uma das novas perspectivas para a profilaxia da candidíase invasiva e sepse de início tardio é pautada no uso de probióticos. De acordo com a Organização Mundial de Saúde (WHO) e a Organização das Nações Unidas para a Alimentação e Agricultura (FDA), os probióticos são microrganismos vivos que, quando administrados em quantidades adequadas, oferecem uma série de benefícios para a saúde do hospedeiro (Organization, 2002). Dentre os principais gêneros estudados, destacam-se Lactobacillus, Bifidobacterium, Propionibacterium e Streptococcus.

Existem várias evidências de que os probióticos modulam a microbiota intestinal e genital, tendo como mecanismos de ação a manutenção da integridade da mucosa, imunomodulação, exclusão de patógenos via competição por proteínas de adesão e nutrientes, além da secreção de moléculas antimicrobianas (de Barros, 2017). Como exemplo, os probióticos podem estimular o sistema imune do hospedeiro a produzir interleucinas pró-inflamatórias TNF- $\alpha$, IL-1b, IL-6 e IL-17, que são importantes no sentido de tornar o sistema imunológico mais competente contra infecções por C.albicans (Marranzino, Villena, Salva, \& Alvarez, 2012; de Oliveira et al., 2017; Leão et al., 2018; Li, Liu, Zhang, Chen, \& Wang, 2019;).

Diversos estudos também já comprovaram que os probióticos produzem ácidos, bacteriocinas, biosurfactantes, peróxido de hidrogênio e moléculas com propriedades de biosurfactantes que afetam a adesão inicial de C.albicans e inibem a formação de biofilmes. Essas propriedades foram descritas in vitro e in vivo (modelo em invertebrados e camundongos), mas, acredita-se que possam ser aplicadas a humanos no sentido preventivo ou terapêutico (Spurbeck \& Arvidson, 2010; Köhler, Assefa, \& Reid, 2012; Orsi et al., 2014; Sabia et al., 2014; Ceresa et al., 2015).

É importante ressaltar que os probióticos devem ser utilizados com precaução em pacientes imunocomprometidos e recém-nascidos prematuros (May \& So, 2014; Khurshid et al., 2015; Szajewska, 2016). Apesar dos benefícios, o uso contínuo de probióticos está relacionado com alguns efeitos adversos, tais como distensão abdominal e flatulência, embora sejam tipicamente moderados e passageiros (Cruchet et al., 2015). Estudos apontam que Lactobacillus spp. e Saccharomyces spp. podem migrar do intestino para a corrente sanguínea, causando bacteremia e fungemia em recém-nascidos, crianças imunodeprimidas ou sob uso de cateter venoso central (May \& So, 2014; Cruchet et al., 2015; Khurshid et al., 2015).

Oncel et al. (2015) compararam o efeito profilático do Lactobacillus reuteri e nistatina em relação à colonização e infeção por Candida em recém-nascidos de baixo peso e chegaram à conclusão que o probiótico foi tão efetivo quanto o antifúngico. Adicionalmente, o grupo suplementado com probióticos obteve uma menor incidência de sepse, hospitalização e intolerâncias alimentares quando comparados com o grupo nistatina (Oncel et al., 2015).

Uma meta-análise realizada por Rao et al. (2016) que avaliou o efeito dos probióticos com grupos placebo e controle em bebês prematuros, constatou que esses microrganismos reduziram significativamente o risco de sepse de início tardio (Rao, Athalye-Jape, Deshpande, Simmer, \& Patole, 2016). Outra meta-análise realizada por Zhang et al. (2016) também relatou significativa redução na sepse fúngica com suplementação de probióticos enteral em neonatos prematuros com sepse de início 
tardio (Zhang, Hu, Liu, Shakya, \& Li, 2016).

\subsection{Candidemia Neonatal}

\subsubsection{Etiopatogenia}

A candidemia se caracteriza pela presença de espécies Candida spp. no sangue, sendo a principal causa de infecções fúngicas invasivas nas crianças hospitalizadas e uma das infecções nosocomiais de corrente sanguínea mais comum. Os principais agentes causadores de candidemia são C.albicans, C.tropicalis, C.parapsilosis, C.glabrata e Pichia kudriavzveii (C.krusei) (Hallen-Adams \& Suhr, 2017; Lamoth, Lockhart, Berkow \& Calandra, 2018).

A espécie C.albicans é conhecida, historicamente, por ser a causa mais prevalente em quadros de candidemia, porém, com a mudança no cenário da epidemiologia, mostra-se o aumento da prevalência de espécies não- albicans de Candida (NAC) (Lamoth et al., 2018).

Cada espécie NAC está associada a condições clínicas específicas, de modo que algumas das espécies NAC, como C.glabrata (Healey \& Perlin, 2018) e C.krusei (Berkow \& Lockhart, 2017) possuem resistência aos azóis e equinocandinas (Healey \& Perlin, 2018), antifúngicos de primeira linha recomendado para o tratamento da candidemia (Pappas et al., 2016).

Os mecanismos de resistência aos azóis em C.albicans, C.parapsilosis e C.tropicalis se baseiam nas substituições específicas de aminoácidos no ERG11, que resulta em uma menor afinidade ao alvo da droga, além de superexpressão de bombas de efluxo (Berkow \& Lockhart, 2017).

Já a espécie C.parapsilosis, é um importante patógeno oportunista. Seu surgimento pode estar relacionado à capacidade de proliferar em soluções que contêm glicose e de colonizar a pele. É responsável por patologias em imunocomprometidos e recém-nascidos com baixo peso ao nascer (Bredt, 2019).

\subsubsection{Epidemiologia}

Ao longo das últimas décadas a distribuição das espécies causadoras de candidemia tem mudado, com redução de C.albicans e aumento na proporção dos complexos de C.glabrata e C.parapsilosis, principalmente. A mudança na epidemiologia em relação às espécies de C.albicans e Candida não albicans é relevante clinicamente e tem impacto direto na resistência a antifúngicos como o fluconazol (Lamoth et al., 2018).

No continente Europeu, a candidemia representa a segunda maior causa de mortalidade dentro do grupo das enfermidades infecciosas em crianças hospitalizadas, de modo que em crianças com comorbidades, a taxa de incidência está de 5 em 1.000 (Gokcebay et al., 2016). No Brasil, um estudo realizado em 16 hospitais mostrou que Candida spp. ficou em sétimo lugar em prevalência e a taxa de mortalidade foi de 72,2 \% (Doi et al., 2016).

A candidemia é relatada entre a sétima e décima infecção da corrente sanguínea mais comum, e considerada a quarta mais frequente em Unidades de Terapia Intensiva (Pana,Roilides, Warris, Groll \& Zaoutis, 2017). As taxas de incidência vêm aumentando devido às comorbidades ou se apresentam estáveis em algumas regiões em função das melhorias de higiene e no manejo da doença (Mccarty \& Pappas, 2016; Epelbaum \& Chasan, 2017).

As infecções invasivas por Candida ocorrem de 10-20 vezes mais quando a criança necessita da UTI; isso ocorre pelo setor ser um ambiente com características e dinâmicas particulares (Epelbaum \& Chasan, 2017). De acordo com um estudo global de Prevalência Estendida em Terapia Intensiva (“Extended Prevalence of Infection in Intensive Care”, EPIC II) Candida spp. representam a terceira causa mais frequente de infecção em UTIs no mundo todo (Lamoth et al., 2018).

Esse mesmo estudo foi responsável por estimar a incidência das espécies de Candida isoladas a partir de infecções da corrente sanguínea em 1265 UTIs de 79 países. Verificou-se a frequência de 6,87 casos em cada 1000 pacientes internados, sendo C.albicans mais frequente $(70,7 \%)$ levando em consideração todos os continentes analisados. Dessa forma, a maior 
incidência desta espécie foi verificada na Europa (71,7\%), enquanto a menor foi na América Latina $(57,1 \%)$ (Lamoth et al., 2018).

É importante ressaltar também que Candida spp. vem sendo relatada como importante patógeno relacionada com a doença fúngica no choque séptico, acometendo de 8 a 10\% desses pacientes (Martin, Mannino, Eaton, \& Moss, 2003; Payen et al., 2015; Bassetti, Vena \& Russo, 2018). Observa-se também que entre os pacientes que desenvolvem choque séptico, essa infecção é considerada a condição clinica com maior taxa de mortalidade, variando de 54 a 66\% (Bassetti et al., 2014; Kollef et al., 2012; Bassetti et al., 2019).

Na Colômbia, a candidemia é relativamente frequente, apresentando maiores taxas de incidência e prevalência se comparada a outras partes do mundo; é a quinta causa de infecção da corrente sanguínea em unidades de terapia intensiva e responde por $88 \%$ das infecções fúngicas em pacientes hospitalizados, com mortalidade variando entre 36 e $40 \%$ (Cortés, Ruiz, Melgarejo-moreno \& Lemos, 2020).

Um total de 316 casos de candidemia foram diagnosticados durante um período de 1 ano em um hospital público no norte da Índia. A maioria (58,8\%) dos pacientes eram menores de 18 anos de idade, enquanto os adultos constituíam 41,2\% de casos. Entre o grupo pediátrico, 74,7\% eram neonatos. Na maioria dos casos, C.krusei (30,3\%) foi o isolado mais comum, seguido por C.tropicalis $(27,8 \%$,$) , C.albicans (14,8 \%)$ e C.pelliculosa $(9,8 \%)$. Na população pediátrica (n =186), C.krusei (44\%) foi a cepa mais encontrada, seguida por C.tropicalis (19,4\%), C.pelliculosa (14,5\%),C.albicans (8,6\%), C.parapsilosis (4,3\%), C.guilliermondii (2,2\%), K. ohmeri (1,6\%), C.ortopsilose (1\%), T. asahii (1\%), Cyberlindnera jadinii (1\%), C.auris $(0,5 \%)$, L. elongisporus (0,5\%), C.fabianii (0,5\%) e C.glabrata (0,5\%) (Kaur et al., 2020).

A fungemia por C.krusei afetou principalmente o grupo pediátrico (44\%), sendo comparado com os adultos (10,8\%). A idade mediana dos pacientes pediátricos foi de 13 dias, tendo em sua maioria o sexo masculino (70,7\%). Entre os 82 casos de C.krusei, 65 (79,3\%) eram neonatos e 72,3\% deles eram do sexo masculino. A idade mediana dos neonatos foi de 9 dias. Apenas $13,8 \%$ desses eram prematuros. Os dados de mortalidade hospitalar disponíveis mostraram mortalidade de 22,94\%. A maioria dos casos foi observada nos meses de setembro e outubro de 2014. O máximo de casos notificados foi da Unidade Neonatal de Emergência Pediátrica (73,1\%), seguido por enfermarias de medicina pediátrica $(15,9 \%)$, UTI de cirurgia pediátrica $(7,3 \%)$, UTI de medicina pediátrica $(2,4 \%)$ e enfermaria de cirurgia pediátrica (1,2\%) (Kaur et al., 2020).

Durante o período do estudo em um hospital infantil em Boston, nos Estados Unidos, houve uma diminuição de 16\% ao ano na taxa de incidência anual de infecções da corrente sanguínea por Candida (CBSI). Bebês com $<1$ ano de idade e crianças entre 1 a 4 anos tiveram as maiores taxas de incidência de CBSI, 1,77 e 2,58 / 1000 pacientes-dia, respectivamente. Não houve declínio na incidência anual de CBSI em pacientes com mais de 4 anos. Também não houve declínio na incidência anual de Candida por infecção da corrente sanguínea associada a um cateter central (CLABSI) entre 2011-2016 (Piqueras et al., 2021).

\subsubsection{Fatores de virulência}

A patogenicidade das espécies de Candida é atribuída a diversos fatores, tais como a produção de biofilme, secreção de enzimas hidrolíticas (sendo a principal a fosfolipase) e a capacidade de evitar as defesas do hospedeiro por formas filamentosas (Dabiri; Shams-Ghahfarokhi; Razzaghi-Abyaneh, 2018; Toth, R., Toth, A., Vagvolgyi, Gacser, 2017; Pharkjaksu, Chongtrakool, Suwannakarn \& Ngamskulrungroj, 2018). Em C.parapsilosis é possível observar também uma menor suscetibilidade a alguns antifúngicos como fluconazol e o aparecimento de estirpes multirresistentes (Prigent et al., 2017).

Em Candida spp. a produção de SAPs (aspartil proteinase) é considerada um dos fatores de virulência de maior importância, pois é responsável por degradar várias proteínas humanas, como hemoglobina, albumina, imunoglobulina secretora A e proteínas da pele. Essa ação proteolítica é responsável pela invasão e penetração tecidual (Pandey, Gupta \& 
Tilak, 2018).

O pleomorfismo (que consiste na capacidade de transição do fungo entre as formas de levedura, hifas ou pseudohifas), é outro fator de virulência de destaque na patogênese da espécie C.albicans (Epelbaum \& Chasan, 2017). Por outro lado, C.tropicalis é caracterizada pela produção expressiva de biofilme, adesão a células epiteliais e endoteliais bucais e comutação fenotípica de levedura para hifa (transição bud-to-hyphae) (Zuza-Alves, Silva-Rocha \& Chaves, 2017).

\subsubsection{Fatores de risco}

O sistema imunológico imaturo dos recém-nascidos associado com a realização de procedimentos médicos invasivos constituem um importante fator de risco para o desenvolvimento de infecções hospitalares. O avanço tecnológico na Medicina tem proporcionando o aumento da sobrevida dos prematuros de peso menor que 1000 gramas, mas, concomitantemente, tem elevado os índices de infecções fúngicas. Neonatos e prematuros com extremo baixo peso ao nascer submetidos a intubação orotraqueal, ventilação mecânica, ao uso de nutrição parenteral, cateteres centrais e administração de medicamentos como corticoides, surfactantes e antibióticos apresentam uma probabilidade 10 vezes maior de desenvolver infecção por Candida no seu primeiro ano de vida (Fu, Wang, Wei, Jiang \& Chen, 2016).

Os fatores de risco relacionados ao desenvolvimento da candidíase e candidemia neonatal também podem apresentar relação a causas maternas (parto vaginal; antibioticoterapia prévia prolongada/inadequada) e ao recém-nascido (prematuridade < 37 semanas) (Sousa, 2019). É importante notar que o transplante de órgão sólido (TOS) também é um importante fator de risco para a C/CI. No estudo realizado por Saxena et al. (2018), dentre 584 pacientes pediátricos submetidos a SOT, 13 desenvolveram candidíase. Além disso, obteve-se a incidência geral de 2,2\% para qualquer doença fúngica invasiva (DFI) (14,3 eventos por 100.000 pacientes/dia), dos quais $86 \%$ pertencia a candidíase (Saxena et al., 2018).

Um estudo realizado por Hassan et al. (2019), destacou que os principais fatores de risco relacionados ao desenvolvimento da candidemia foram uso de antibióticos (fluoroquinolonas) e profilaxia antifúngica com fluconazol (Ericson et al., 2016). Por outro lado, o cateterismo venoso prolongado e a ventilação invasiva apresentaram correlação mais estreita com o desenvolvimento da candidemia não-albicans (Hassan, Yousef, Abu Elhamed, Ali \& Madkour, 2019).

Ademais, Chen et al. (2016), publicaram um estudo que demonstrou que a nutrição parenteral total (NPT) e cateterismo venoso central foram os principais fatores predisponentes para candidemia (Chen et al., 2016). Por outro lado, Yadav, Dahiya e Budhani (2017) afirmaram que o uso prolongado de antibióticos, a prematuridade e o extremo baixo peso ao nascer são fatores de risco significativos.

Um estudo realizado em um hospital universitário mostrou que os fatores de risco significativos para mortalidade em pacientes com candidemia foram: a necessidade de procedimentos invasivos (ventilação mecânica, intubação orotraqueal e hemodiálise), uso de corticosteroides e infecção por C.parapsilosis, sendo as espécies não C.albicans os agentes causadores de candidemia mais prevalentes. Esse estudo também destacou a importância do tempo total de internação, a necessidade de cuidados relacionados aos procedimentos invasivos e de ações para contribuir com a melhora da imunidade do paciente, como um bom equilíbrio nutricional, que contribuirá para a diminuição da gravidade das infecções por Candida e sua morbimortalidade (Alves et al., 2020).

Os fatores de risco observados nos casos de candidemia por C.krusei incluíram: doença gastrointestinal e uso prévio de antibióticos (principalmente carbapenêmicos), quando comparados aos casos de candidemia por $C$.pelliculosa. Presença de malformações congênitas, admissão na UTI e ventilação mecânica, também são fatores de risco observados na candidemia por C.tropicalis. A taxa de mortalidade hospitalar foi significativamente menor entre pacientes com candidemia por C.krusei (23\%) em comparação com pacientes infectados por C.tropicalis (42,9\%). Além disso, o tempo para a positividade da hemocultura para C.krusei foi menor em comparação com a C.tropicalis (Kaur et al., 2020). 
Os fatores de risco maternos associados ao desenvolvimento de candidemia foram analisados em um estudo em Kerala, observando aumento do risco no parto vaginal e mães que receberam esteroides pré-natais. Entre o risco neonatal, fatores como prematuridade, baixo peso ao nascer, asfixia ao nascer, ressuscitação (que precisam de bolsa e máscara), alimentação enteral retardada > 24 horas e a hiperglicemia neonatal foram significativamente associadas à candidemia neonatal. Fatores de risco hospitalar associados a candidemia neonatal foram: infusão de aminoácidos, esteróides, uso de mais de dois antibióticos, acesso venoso central, intubação traqueal, ventilação assistida, oxigênio, imunoglobulina intravenosa, cafeína e surfactante. Muitos desses fatores de risco são interdependentes. Os fatores de risco independentes foram: mais de dois antibióticos, infusão de aminoácidos, hiperglicemia neonatal antes da hemocultura e candidíase vaginal materna (Kr, Daniel \& Philip, 2020).

\subsubsection{Manifestações Clínicas}

As manifestações clínicas mais comuns são dor abdominal, febre, vômito, edema, hemorragia gastrointestinal e pulmonar e falência de múltiplos órgãos (Liu et al., 2015). A febre pode se apresentar alta $39-40^{\circ} \mathrm{C}$, moderada ou simples, podendo causar até uma hipotermia no infectado. Além disso, pode-se ter presença de taquicardia persistente, crises de calafrios, rash cutâneo no tronco e nas extremidades, simulando uma reação anafilática (Sierra, 2009). A sepse também é uma das manifestações que se apresenta em muitos casos, sendo um dos grandes fatores da mortalidade. Outra característica clínica importante é o comprometimento do desenvolvimento neurológico (Chen et al., 2016).

Um estudo realizado em Kelara constatou que os sintomas e sinais como letargia (67\%), angústia respiratória (67\%), apneia $(35,1 \%)$, hipoxemia silenciosa $(21,3 \%)$, convulsão (14,9\%), intolerância alimentar (33\%), tolerância alimentar abaixo do limite (16\%), choro fraco (86,2\%), infecção da mucosa por Candida (5,3\%), distensão abdominal (54,3\%) e hepatoesplenomegalia $(22,3 \%)$ foram significativamente maior nos casos em comparação com os controles. Bebês recém-nascido com Candida mostraram um aumento na proporção de trombocitopenia $(75,5 \%)$, proteína C reativa positiva (47,9\%) e Líquido Cefalorraquiano anormal $(9,6 \%)(\mathrm{Kr}$ et al., 2020).

\subsubsection{Diagnóstico}

O diagnóstico da candidíase inicia com a avaliação das manifestações clínicas no paciente. Dentre elas é possível elencar a endoftalmite (lesões coriorretinianas e vítreas), candidíase hepatoesplênica (candidíase disseminada crônica, febre persistente, dor abdominal nos quadrantes superior esquerdo ou superior direito e anorexia), meningoencefalite hematogênica por Candida (HCME) (convulsões, hemorragias intraventriculares, atrasos ou regressão do desenvolvimento e pleocitose do LCR), candidíase renal (diminuição da depuração da creatinina, nefropatia obstrutiva), endocardite (sopros, manifestações embólicas periféricas e insuficiência cardíaca congestiva), infecções osteoarticulares (lesões osteoarticulares que não respondem a terapia antibacteriana) e candidíase aguda disseminada (lesões cutâneas múltiplas, mialgias difusas, hipotensão e insuficiência renal) (Walsh, Katragkou, Chen, Salvatore \& Roilides, 2019).

No diagnóstico laboratorial, a hemocultura é pouco sensível, sendo assim não é o método de escolha para detectar a candidíase invasiva. Dessa forma, novos métodos estão sendo utilizados, como imunoensaios enzimáticos para mananas circulantes, sistemas enzimáticos para deteção de $(1 \rightarrow 3)$ - $\beta$ - D- glucano e sistemas de amplificação de ácido nucleico (Huppler, Fisher, Lehrnbecher, Walsh \& Steinbach, 2017; Mccarthy \& Walsh, 2017; Hamula et al., 2016; Mylonakis et al., 2015; Clancy et al., 2018).

Outro sistema recentemente licenciado pelo FDA dos EUA que vem sendo utilizado como método superior aos sistemas convencionais para a detecção de candidemia é o painel T2Candida, que utiliza um T2 magnético (tecnologia de ressonância), juntamente com a amplificação do ácido nucleico derivado dos patógenos para identificar as cinco espécies alvo 
mais causadoras de candidemia em torno de 2 a 5 horas (Mylonakis et al., 2015; Clancy et al, 2018 ).

Tem-se também um importante biomarcador para detecção e monitoramento terapêutico de infecções por Candida spp. no Sistema Nervoso Central, o Cerebrospinal Fluid (CSF) $(1 \rightarrow 3)$ - $\beta$ - D- Glucano. Em um estudo realizado por Salvatore, Chen e colegas, ocorreu a medição dos níveis de $(1 \rightarrow 3)$ - $\beta$ - D- glucano em amostras coletadas de LCR de pacientes pediátricos, com o provável diagnóstico de Candida hematogênica meningoencefalite (HCME) ou o diagnóstico conclusivo de aspergilose do Sistema Nervoso Central (SNC) (Salvatore et al., 2016 ) . Entre os nove casos de infecção fúngica do SNC, sete foram causados pela HCME. No começo do estudo, todos os pacientes tinham $(1 \rightarrow 3)$ - $\beta$ - D- glucano detectável em seu LCR. Nos seis pacientes que completaram a terapia para HCME, houve uma queda nos níveis elevados de LCR ( $1 \rightarrow$ 3) $-\beta$ - D- glucano, diminuindo para $<31 \mathrm{pg} / \mathrm{mL}$.

O monitoramento dos níveis de CSF em série $(1 \rightarrow 3)$ - $\beta$ - D- glucano no HCME, foi de suma importância para determinar a duração da terapia, que variou entre 3 a 6 meses, com base nas avaliações individuais. $O$ estudo confirmou a utilidade da medição dos níveis de $(1 \rightarrow 3)$ - $\beta$ - D- glucano no LCR para diagnóstico inicial e monitoramento terapêutico de HCME (Ceccarelli et al., 2016; Mccarthy \& Walsh, 2017; Farrugia et al., 2016).

\subsubsection{Tratamento}

A candidemia requer tratamento antifúngico por várias semanas; o tipo da medicação e a duração dependem de fatores específicos do paciente, como comorbidades, idade, estado imunológico, gravidade de infecção e sensibilidade das espécies identificadas (Centers for Disease Control and Prevention, 2019). Existe diferentes antifúngicos disponíveis para o tratamento da candidíase (anfotericina B, flucitosina, azóis), entretanto, foi observado alguns efeitos adversos desses medicamentos, devido a toxicidade ou interações medicamentosas. Mais recentemente, as equinocandinas foram introduzidas (anidulafungina, caspofungina e micafungina) e são fungicidas bem tolerados que possuem amplo espectro de ação com maiores taxas de sucesso clínico (Pappas et al., 2016).

Outra medida utilizada é a terapia probiótica, que é baseada na administração de bactérias não patogênicas para deslocar os organismos patogênicos do intestino (Matsubara, Bandara, Mayer \& Samaranayake, 2016). Essa terapia é muito utilizada para reduzir a colonização de Candida spp nos neonatos prematuros (Manzoni, 2007).

Dentre as drogas utilizadas para o tratamento, encontram-se as classes: poliênicos (anfotericina B), azóis (Ericson et al., 2016) e equinocandinas (anidulafungina, micafungina e caspofungina) (Bredt, 2017). A Sociedade Europeia de Microbiologia Clínica e Doenças Infecciosas recomenda o uso das equinocandinas seguida da terapia de redução com a administração de fluconazol ou voricozol entre 5-7 dias em pacientes que apresentaram melhora (Pappas et al., 2016).

Em um estudo realizado na China com o objetivo de identificar cepas resistentes às drogas antifúngicas, constatou-se que C.parapsilosis foi suscetível ao fluconazol (94,4\% susceptibilidade), posaconazol (97,5\%) voriconazol (95,3\% suscetível) e itraconazol (100\%), sendo que entre a população de estudo, dezesseis isolados resistentes ao fluconazol eram nosocomiais. Todos os 319 isolados eram suscetíveis a equinocandinas, exceto dois que foram classificados como moderadamente suscetíveis a micafungina (Zhang et al., 2020).

Ao se realizar o teste de sensibilidade para anfotericina B (Papadimitriou-Olivgeris et al., 2019) em 115 isolados, caspofungina e voriconazol em 114 isolados, fluconazol em 112 isolados e para micafungina em 89 isolados, foi observado que todos os microrganismos testados foram sensíveis a $\mathrm{AmB}$ e voriconazol. A caspofungina e a micafungina mostraram excelente ação contra a maioria das espécies de Candida, incluindo todos os isolados de C.parapsilosis. Quase todos os isolados foram sensíveis ao fluconazol (96,7\%). A resistência à micafungina foi observada em dois isolados de C.glabrata, em um paciente com episódios anteriores de infecções da corrente sanguínea por Candida (CBSI) tratado com esse antifúngico. Não foi encontrada multirresistência em nenhum dos isolados testados. Geralmente a droga mais prescrita foi o AmB 
lipossomal, usado principalmente em neonatos e empiricamente em pacientes neutropênicos até que fosse excluído o envolvimento do sistema nervoso central (Piqueras et al., 2021).

\subsubsection{Prevenção}

A prevenção da candidemia baseia-se nos fatores de risco para aquisição da infecção. Em relação ao paciente, este deve ter uma exposição mínima aos antibióticos de amplo espectro assim como ao uso de cateteres e dispositivos invasivos, devendo ser reavaliada diariamente, a fim de diminuir o risco de infecção. (Kothavade, Kura, Valand \& Panthaki, 2010; Vallabhaneni et al., 2015).

Em 2015, Fisher e cols. publicaram um estudo sobre retenção de cateter e mortalidade em crianças. Avaliaram 285 pacientes com Candida invasiva e presença de cateter na Filadélfia, sendo a C.albicans a espécie mais encontrada, seguida da C.parapsilosis e C.tropicalis. A retenção do cateter associou-se ao risco aumentado de mortalidade. (Fischer et al., 2016).

Com isso, a Sociedade Americana de Doenças Infecciosas recomenda a remoção do cateter quando houver suspeita de que ele seja a fonte de infecção (Pappas et al., 2016).

No que se refere ao risco de aquisição nosocomial, a medida mais efetiva e barata para evitar as infecções hospitalares, é sem dúvida a higienização das mãos. A Organização Pan-Americana de Saúde (OPAS) faz várias recomendações quando tem casos de $C$.auris, como fazer a limpeza e manutenção do ambiente e equipamentos, cuidados na retirada dos pacientes infectados, lavagem das vestimentas dos pacientes em ciclos separados dos demais (Paho, 2016).

Além disso, outra medida preventiva é através da profilaxia medicamentosa, em que se aplica em algumas situações específicas, como em pacientes transplantados, pacientes de baixo peso na neonatologia, apesar de controvérsia a respeito da duração da profilaxia (Kaur \& Chakrabarti, 2017).

\subsubsection{Estratégias para redução da morbimortalidade na candidemia}

Existem vários desafios que devem ser enfrentados no manejo dos casos de candidemia os quais incluem a indisponibilidade de testes diagnósticos avançados e drogas antifúngicas em muitas áreas; práticas de saúde e controle de infecção; falta de conhecimento sobre infecções fúngicas; uso indevido e exacerbado de antibióticos; baixa adesão à terapia antifúngica e programas de manejo. Considerando as limitações acima, algumas estratégias podem ser utilizadas para diminuir a taxa de mortalidade em pacientes com candidemia (Kaur \& Chakrabarti, 2017).

1. Aprimorar o diagnóstico: Embora os marcadores sorológicos fúngicos específicos sejam caros, existem alternativas mais baratas, como PCT e PCR, que têm mostrado um alto valor preditivo negativo, que podem ser padronizadas e validadas para excluir candidemia. Além disso, é importante uma comunicação acessível entre o laboratório e os médicos, para que o resultado dos exames seja repassado de forma rápida, podendo assim, iniciar uma terapia antifúngica precoce. Ademais, devese ter laboratórios de emergência funcionando 24 horas por dia, equipados com sistemas automatizados de hemocultura, com os resultados sendo comunicados ao médico em tempo real (Kaur \& Chakrabarti, 2017).

2. Conhecimento: é de grande importância que os profissionais da saúde tenham conhecimento em relação as práticas de prevenção de infecções, e saibam fazer um diagnóstico precoce da candidemia. Com isso, o papel das palestras, treinamentos práticos, e o autoestudo para médicos e equipe de enfermagem são indispensáveis para uma diminuição significativa nas taxas de infecção da corrente sanguínea associada ao cateter (CLABSI) (Kaur \& Chakrabarti, 2017).

3.Controle de origem da infecção: deve ser implementado para controle do foco da infecção e assim reduzir as condições favoráveis que promovem o crescimento dos microrganismos ou que mantêm o comprometimento das defesas do hospedeiro (Lagunes et al., 2017). Com isso, recomenda-se a remoção dos cateteres venosos centrais (CVCs) mais brevemente possível na candidemia em pacientes não neutropênicos quando acredita-se que a fonte seja o CVC (Pappas et al., 2016 ). Já no 
paciente neutropênico, a decisão dessa remoção deve ser individualizada, pois a origem de Candida neste grupo é geralmente diferente de um CVC (por exemplo, trato gastrointestinal) (Pappas et al., 2016).

4.Epidemiologia: As decisões de tratamento baseiam-se no conhecimento da epidemiologia (frequência de isolamento e suscetibilidade antifúngica de cada espécie de Candida) (Pappas et al., 2016). Além disso, os dados epidemiológicos também ajudarão no planejamento de estratégias locais de manejo da candidemia.

5.Tratamento: $\mathrm{O}$ manejo adequado e precoce do paciente com Candida invasiva é fundamental para reduzir a permanência no hospital ou na UTI, e assim diminuir a mortalidade por candidemia. Lopez-Cortes et al. (2016) realizaram um estudo multicêntrico e observaram que não houve aumento na mortalidade quando a terapia era feita por fluconazol no lugar de equinocandinas; dessa forma, os autores apoiam o uso de fluconazol com segurança (López-Cortés et al., 2016). Tem-se também, o desoxicolato de anfotericina B que pode ser usado como uma alternativa econômica, especialmente em neonatos.

6.Rastreio e descolonização de candidíase vaginal materna: Triagem e manejo da mulher que possui colonização vaginal pela Candida é essencial para prevenir a colonização neonatal em estágio inicial, pois os bebês prematuros são colonizados por Candida da flora materna (Olowe, O, Makanjuola, Olowe, R. \& Adekanle, 2014; Sopian, Sa'adiah Shahabudin, Lung \& Sandai, 2016).

7.Administração de probióticos: Ensaios clínicos realizados em recém-nascidos prematuros demonstraram um efeito benéfico de Saccharomyces boulardii sem apresentar qualquer evidência de fungemia ou sepse (Roy et al., 2017), mas o uso de probióticos deve ser realizado com cautela em neonatos pré-termo e pacientes imunocomprometidos, pois não existem estudos suficientes que comprovem a segurança desse produto (Atici et al., 2017).

8.Contenção da infecção: Chen et al. (2016) relataram a execução de técnicas intensas de higiene das mãos, além da profilaxia com fluconazol, para se obter mais sucesso na prevenção de candidemia em prematuros < 33 semanas em UTIN do que a profilaxia isolada (Chapman et al., 2017).

\subsubsection{Perspectivas futuras: o que há de novo para o tratamento da candidemia?}

Uma nova alternativa não convencional para reduzir a candidemia é o uso da terapia probiótica. Faz-se o consumo oral de bactérias não patogênicas, a fim de deslocar os organismos patogênicos do intestino (Matsubara et al., 2016). Estudos demonstram que os probióticos são capazes de alterar a microbiota intestinal e reduzir o excessivo crescimento de organismos potencialmente patogênicos, sendo capaz de reduzir infecções sistêmicas (Mantadakis et al., 2018).

Kumar et al. fez um estudo no qual avaliaram crianças de 3 meses a 12 anos em uma UTIP de 12 leitos, por dois períodos de 9 meses. Um grupo fez uso de probióticos e o outro não, além do uso de antibióticos de amplo espectro. No estudo, em 1,2 \% das crianças do grupo probiótico apresentou candidemia, comparado com 3,7\% no grupo controle. Além disso, ocorreu candidúria em 10,7\% e 22\% nos grupos de probióticos e controle, respectivamente (Kumar, Bansal, Chakrabarti \& Singhi et al., 2013). No entanto, apesar de alguns estudos realizados, não é possível confirmar com toda certeza se os probióticos realmente diminuem a prevalência de candidemia em pacientes pediátricos (Mantadakis et al., 2018).

\section{Considerações Finais}

Apesar da morbimortalidade da candidíase e candidemia neonatal variar entre diferentes partes do mundo, é necessário a implementação de políticas públicas voltadas para a prevenção e vigilância em saúde. O controle mais rígido dos fatores de risco dentro do ambiente hospitalar associado ao cuidado dos profissionais, além do uso racional de antifúngicos seja na prevenção ou tratamento constituem medidas fundamentais para o manejo adequado dessas patologias. É importante ressaltar também sobre a mudança gradual no curso epidemiológico da candidíase e candidemia neonatal pela emergência de 
novas espécies não albicans que podem representar sério risco para a saúde dos neonatos. Estudos futuros que correlacionem a epidemiologia com a evolução clínica da candidíase e candidemia neonatal são necessários para melhor manejo das patologias.

\section{Agradecimentos}

Os autores agradecem ao Programa Institucional de Bolsas de Iniciação Cientifica da Universidade Ceuma pelo financiamento da pesquisa.

\section{Referências}

Adams-Chapman, I., Bann, C.M., Das, A., Goldberg, R. N., Stoll, B. J., Walsh, M. C., \& Watterberg, K. L. (2013). Neurodevelopmental outcome of extremely low birth weight infants with Candida infection. The Journal of pediatrics, 163(4), 961-967.

Agarwal, R. R., Agarwal, R. L., Chen, X., Lua, J. L., \& Ang, J. Y. (2017). Epidemiology of invasive fungal infections at two tertiary care neonatal intensive care units over a 12-year period (2000-2011). Global pediatric health, 4, $2333794 X 17696684$.

Alves, P. G. V., Melo, S. G. O., Bessa, M. A. d. S., Brito, M. d. O., Menezes, R. d. P., Araújo, L. B. d., \& Röder, D. V. D. d. B. (2020). Risk factors associated with mortality among patients who had candidemia in a university hospital. Revista da Sociedade Brasileira de Medicina Tropical, 53.

Arsenault, A. B., \& Bliss, J. M. (2015). Neonatal candidiasis: new insights into an old problem at a unique host-pathogen interface. Current fungal infection reports, 9(4), 246-252.

Atıcı, S., Soysal, A., Cerit, K. K., Yılmaz, Ş., Aksu, B., Kıyan, G., \& Bakır, M. (2017). Catheter-related Saccharomyces cerevisiae Fungemia Following Saccharomyces boulardii Probiotic Treatment: In a child in intensive care unit and review of the literature. Medical mycology case reports, $15,33-35$.

Auriti, C., Falcone, M., Ronchetti, M. P., Goffredo, B. M., Cairoli, S., Crisafulli, R., \& Pai, M. P. (2016). High-dose micafungin for preterm neonates and infants with invasive and central nervous system candidiasis. Antimicrobial agents and chemotherapy, 60(12), 7333-7339.

Bachur, L. F. (2019). Avaliação dos níveis séricos de 1-3-BD-Glucana e da resposta inflamatória em pacientes sépticos comparativamente a pacientes com candidíase invasiva (Tese de Doutorado). Universidade Estadual de Campinas, Campinas, Brasil.

Bassetti, M., Giacobbe, D. R., Vena, A., Trucchi, C., Ansaldi, F., Antonelli, M., \& Montravers, P. (2019). Incidence and outcome of invasive candidiasis in intensive care units (ICUs) in Europe: results of the EUCANDICU project. Critical Care, 23(1), 1-7.

Bassetti, M., Righi, E., Ansaldi, F., Merelli, M., Cecilia, T., De Pascale, G., \& \& Tumbarello, M. (2014). A multicenter study of septic shock due to candidemia: outcomes and predictors of mortality. Intensive care medicine, 40(6), 839-845.

Bassetti, M., Vena, A., \& Russo, A. (2018). Management of patients with septic shock due to Candida infection. Hospital Practice, $46(5), 258-265$.

Benedict, K., Roy, M., Kabbani, S., Anderson, E. J., Farley, M. M., Harb, S., \& Marceaux, K. (2018). Neonatal and pediatric candidemia: results from population-based active laboratory surveillance in four US locations, 2009-2015. Journal of the Pediatric Infectious Diseases Society, 7(3), e78-e85.

Benjamin, D. K., Stoll, B. J., Fanaroff, A. A., McDonald, S. A., Oh, W., Higgins, R. D., \& Goldberg, R. (2006). Neonatal candidiasis among extremely low birth weight infants: risk factors, mortality rates, and neurodevelopmental outcomes at 18 to 22 months. Pediatrics, 117(1), 84-92.

Benjamin, D. K., Stoll, B. J., Gantz, M. G., Walsh, M. C., Sánchez, P. J., Das, A., \& Miller, N. A. (2010). Neonatal candidiasis: epidemiology, risk factors, and clinical judgment. Pediatrics, 126(4), e865-e873.

Berkow, E. L. \& Lockhart, S. R. (2017). Fluconazole resistance in Candida species: a current perspective. Infection and drug resistance, 10 , p. 237.

Blyth, C. C., Chen, S. C., Slavin, M. A., Serena, C., Nguyen, Q., Marriott, D., \& Sorrell, T. C.(2009). Not just little adults: candidemia epidemiology, molecular characterization, and antifungal susceptibility in neonatal and pediatric patients. Pediatrics, 123(5), 1360-1368.

Boo, N. Y., \& Cheah, I. G. S. (2016). Factors associated with inter-institutional variations in sepsis rates of very-low-birth-weight infants in 34 Malaysian neonatal intensive care units. Singapore medical journal, 57(3), 144.

Bredt, C. S. D. O. (2017). Estudo multicêntrico de candidemias em oito hospitais no estado do Paraná: rede candidemia Paraná (2016-2017) (Tese de Doutorado). Universidade Federal do Paraná, Curitiba, Brasil.

Brühwasser, C., Hinterberger, G., Mutschlechner, W., Kaltseis, J., Lass-Flörl, C., \& Mayr, A. (2016). A point prevalence survey on hand hygiene, with a special focus on Candida species. American journal of infection control, 44(1), 71-73.

Cailes, B., Kortsalioudaki, C., Buttery, J., Pattnayak, S., Greenough, A., Matthes, J., \& Heath, P. T. (2018). Epidemiology of UK neonatal infections: the neonIN infection surveillance network. Archives of Disease in Childhood-Fetal and Neonatal Edition, 103(6), F547-F553.

Calley, J. L., \& Warris, A. (2017). Recognition and diagnosis of invasive fungal infections in neonates. Journal of Infection, 74, S108-S113.

Camp, I., Spettel, K., \& Willinger, B. (2020). Molecular Methods for the Diagnosis of Invasive Candidiasis. Journal of Fungi, 6(3), 101.

Canassa, A. L., \& da Cruz, D. T. (2019). Incidência e perfil de suscetibilidade de candidemias de um hospital público em Mato Grosso do Sul, Brasil. Revista Brasileira de Pesquisa em Saúde/Brazilian Journal of Health Research, 21(4), 110-117. 
Cantey, J. B., \& Milstone, A. M. (2015). Bloodstream infections: epidemiology and resistance. Clinics in perinatology, 42(1), 1-16.

Ceccarelli, G., Ghezzi, M. C., Raponi, G., Brunetti, G., Marsiglia, C., Fallani, S., ... \& Venditti, M. (2016). Voriconazole treatment of Candida tropicalis meningitis: Persistence of $(1,3)-\beta$-D-glucan in the cerebrospinal fluid is a marker of clinical and microbiological failure: A case report. Medicine, $95(31)$.

Centers for Disease Control and Prevention. (2019). Invasive candidiasis. https://www.cdc.gov/fungal/diseases/candidiasis/invasive/index.html

Ceresa, C., Tessarolo, F., Caola, I., Nollo, G., Cavallo, M., Rinaldi, M., \& Fracchia, L. (2015). Inhibition of Candida albicans adhesion on medical-grade silicone by a Lactobacillus-derived biosurfactant. Journal of applied microbiology, 118(5), 1116-1125.

Chang, Y. J., Choi, I. R., Shin, W. S., Lee, J. H., Kim, Y. K., \& Park, M. S. (2013). The control of invasive Candida infection in very low birth weight infants by reduction in the use of 3rd generation cephalosporin. Korean journal of pediatrics, $56(2), 68$.

Chapman, B., Slavin, M., Marriott, D., Halliday, C., Kidd, S., Arthur, I., \& Chen, S. (2017). Changing epidemiology of Candidaemia in Australia. Journal of Antimicrobial Chemotherapy, 72(4), 1103-1108.

Chen, J., Jiang, Y., Wei, B., Ding, Y., Xu, S., Qin, P., \& Fu, J. (2016). Epidemiology of and risk factors for neonatal candidemia at a tertiary care hospital in western China. BMC infectious diseases, 16(1), 1-5.

Clancy, C.J., Pappas, P. G., Vazquez, J., Judson, M. A., Kontoyiannis, D. P., Thompson III, G. R., ... \& Caliendo, A. M. (2018). Detecting infections rapidly and easily for candidemia trial, part 2 (DIRECT2): a prospective, multicenter study of the T2Candida panel. Clinical Infectious Diseases, 66(11), $1678-1686$.

Cortés, J. A., Ruiz, J. F., Melgarejo-Moreno, L. N., \& Lemos, E. V. (2020). Candidemia in Colombia. Biomédica, $40(1), 195-207$.

Cruchet, S., Furnes, R., Maruy, A., Hebel, E., Palacios, J., Medina, F., \& Sdepanian, V. (2015). The use of probiotics in pediatric gastroenterology: a review of the literature and recommendations by Latin-American experts. Pediatric Drugs, 17(3), 199-216.

da Silva Rios, J. F., \& de Castro Romanelli, R. M. (2016). Candidíase invasiva em unidade neonatal. Journal of Infection Control, 5(1).

Dabiri, S., Shams-Ghahfarokhi, M., \& Razzaghi-Abyaneh, M. (2018). Comparative analysis of proteinase, phospholipase, hydrophobicity and biofilm forming ability in Candida species isolated from clinical specimens. Journal de Mycologie MÚdicale, 28(3), 437-442.

Davidson, L., Netea, M. G., \& Kullberg, B. J. (2018). Patient susceptibility to candidiasis — a potential for adjunctive immunotherapy. Journal of Fungi, 4(1), 9.

de Barros, A. M. F. R. (2017). Probióticos em Idade Pediátrica: Riscos, Benefícios e Recomendações (Dissertação de Mestrado). Universidade do Porto, Porto, Portugal.

De Bernardis, F., Graziani, S., Tirelli, F., \& Antonopoulou, S. (2018). Candida vaginitis: virulence, host response and vaccine prospects. Medical mycology, 56(suppl_1), S26-S31.

de Melo Riceto, É. B., de Paula Menezes, R., Penatti, M. P. A., \& dos Santos Pedroso, R. (2015). Enzymatic and hemolytic activity in different Candida species. Revista iberoamericana de micologia, 32(2), 79-82.

de Oliveira, F. E., Rossoni, R. D., de Barros, P. P., Begnini, B. E., Junqueira, J. C., Jorge, A. O. C., \& de Oliveira, L. D. (2017). Immunomodulatory effects and anti-Candida activity of lactobacilli in macrophages and in invertebrate model of Galleria mellonella. Microbial pathogenesis, $110,603-611$.

de Paula Menezes, R., Silva, F. F., Melo, S. G., Alves, P. G., Brito, M. O., de Souza Bessa, M. A., \& Röder, D. v. D. (2019). Characterization of Candida species isolated from the hands of the healthcare workers in the neonatal intensive care unit. Medical mycology, 57(5), 588-594.

Deorukhkar, S. C., \& Saini, S. (2015). Non albicans Candida species: a review of epidemiology, pathogenicity and antifungal resistance. Pravara Medical Review, 7(3), 7-15.

Doi, A. M., Pignatari, A. C.C., Edmond, M. B., Marra, A. R., Camargo, L. F. A., Siqueira, R. A., \& Colombo, A. L. (2016). Epidemiology and microbiologic characterization of nosocomial candidemia from a Brazilian national surveillance program. PloS one, 11(1), e0146909.

Dutta, A. (2019). Candidiasis Introduction to Clinical Infectious Diseases (pp. 335-340). Springer, Cham

Epelbaum, O., \& Chasan, R. (2017). Candidemia in the Intensive Care Unit.. Clin Chest Med, 38(3), 493-509. https://www.sciencedirect.com/science/article/abs/pii/S027252311730045X?via\%3Dihub

Ericson, J. E., Kaufman, D. A., Kicklighter, S. D., Bhatia, J., Testoni, D., Gao, J., \& Simone, G. (2016). Fluconazole prophylaxis for the prevention of candidiasis in premature infants: a meta-analysis using patient-level data. Clinical Infectious Diseases, 63(5), 604-610.

Estrela, C.(2018). Metodologia Científica: Ciência, Ensino, Pesquisa. Artes Médicas.

Farrugia, M. K., Fogha, E. P., Miah, A. R., Yednock, J., Palmer, H. C., \& Guilfoose, J. (2016). Candida meningitis in an immunocompetent patient detected through $(1 \rightarrow 3)$-beta-d-glucan. International Journal of Infectious Diseases, 51, 25-26.

Fisher, B. T., Vendetti, N., Bryan, M., Prasad, P. A., Russell Localio, A., Damianos, A., \& Zaoutis, T. E. (2016). Central venous catheter retention and mortality in children with candidemia: a retrospective cohort analysis. Journal of the Pediatric Infectious Diseases Society, 5(4), 403-408.

França, E. J. G., Furlaneto-Maia, L., \& Furlaneto, M. C.(2017). Hemolytic capability and expression of a putative haem oxygenase-encoding gene by blood isolates of Candida tropicalis are influenced by iron deprivation and the presence of hemoglobin and erythrocytes. Microbial pathogenesis, $105,235-239$. 
Fu, J., Ding, Y., Jiang, Y., Mo, S., Xu, S., \& Qin, P. (2018). Persistent candidemia in very low birth weight neonates: risk factors and clinical significance. BMC infectious diseases, 18(1), 1-6.

Fu, J., Wang, X., Wei, B., Jiang, Y., \& Chen, J. (2016). Risk factors and clinical analysis of candidemia in very-low-birth-weight neonates. American journal of infection control, 44(11), 1321-1325.

Gokcebay, D. G., Yarali, N., Isik, P., Bayram, C., Ozkaya-Parlakay, A., Kara, A., \& Tunç, B. (2016). Candida associated bloodstream infections in pediatric hematology patients: a single center experience. Mediterranean journal of hematology and infectious diseases, $8(1)$.

Greenberg, R. G., \& Benjamin Jr, D. K. (2014). Neonatal candidiasis: diagnosis, prevention, and treatment. Journal of Infection, 69, S19-S22.

Hallen-Adams, H. E. \& Suhr, M. J. (2017). Fungi in the healthy human gastrointestinal tract. Virulence, 8(3), 352-358.

Hamula, C.L., Hughes, K., Fisher, B. T., Zaoutis, T. E., Singh, I. R., \& Velegraki, A. (2016). T2Candida provides rapid and accurate species identification in pediatric cases of candidemia. American journal of clinical pathology, 145(6), 858-861.

Hassan, D. M., Yousef, R. H., Abu Elhamed, W. A., Ali, A. A., \& Madkour, L. A. (2019). Candidemia in the neonatal intensive care unit: Insights on epidemiology and antifungal drug susceptibility patterns. Archives of Pediatric Infectious Diseases, 7(1).

Healey, K. R. \& Perlin, D. S. (2018). Fungal resistance to echinocandins and the MDR phenomenon in Candida glabrata. Journal of Fungi, 4(3), 105.

Hope, W., Castagnola, E., Groll, A., Roilides, E., Akova, M., Arendrup, M., \& Cornely, O. (2012). ESCMID* guideline for the diagnosis and management of Candida diseases 2012: prevention and management of invasive infections in neonates and children caused by Candida spp. Clinical Microbiology and Infection, 18, 38-52.

Hsu, J. F., Lai, M. Y., Lee, C.W., Chu, S. M., Wu, I. H., Huang, H. R., \& Tsai, M. H. (2018). Comparison of the incidence, clinical features and outcomes of invasive candidiasis in children and neonates. BMC infectious diseases, 18(1), 1-11.

Huppler, A. R., Fisher, B. T., Lehrnbecher, T., Walsh, T. J., \& Steinbach, W. J. (2017). Role of molecular biomarkers in the diagnosis of invasive fungal diseases in children. Journal of the Pediatric Infectious Diseases Society, 6, S32-S44.

Kaur, H., \& Chakrabarti, A. (2017). Strategies to reduce mortality in adult and neonatal candidemia in developing countries. Journal of Fungi, 3(3), 41.

Kaur, H., Shankarnarayana, S. A., Hallur, V., Muralidharan, J., Biswal, M., Ghosh, A. K., \& Rudramurthy, S. M. (2020). Prolonged outbreak of Candida krusei candidemia in paediatric ward of tertiary care hospital. Mycopathologia, 185(2), 257-268.

Kelly, M. S., Benjamin, D. K., \& Smith, P. B. (2015). The epidemiology and diagnosis of invasive candidiasis among premature infants. Clinics in perinatology, 42(1), 105-117.

Khurshid, M., Aslam, B., Nisar, M. A., Akbar, R., Rahman, H., Khan, A. A., \& Rasool, M. H. (2015). Bacterial munch for infants: potential pediatric therapeutic interventions of probiotics. Future microbiology, 10(11), 1881-1895.

Kliegman, R. M., Geme, J. S., Blum, N., Shah, S. S., \& Tasker, R. C.(2020). Nelson. Tratado de pediatría. Barcelona, Espanha: Elsevier Health Sciences.

Köhler, G. A., Assefa, S., \& Reid, G. (2012). Probiotic interference of Lactobacillus rhamnosus GR-1 and Lactobacillus reuteri RC-14 with the opportunistic fungal pathogen Candida albicans. Infectious diseases in obstetrics and gynecology, 2012.

Kollef, M., Micek, S., Hampton, N., Doherty, J. A., \& Kumar, A. (2012). Septic shock attributed to Candida infection: importance of empiric therapy and source control. Clinical infectious diseases, 54(12), 1739-1746.

Kothavade, R. J., Kura, M. M., Valand, A. G., \& Panthaki, M. H. (2010). Candida tropicalis: its prevalence, pathogenicity and increasing resistance to fluconazole. Journal of medical microbiology, 59(8), 873-880.

KR, J. S., Daniel, S., \& Philip, R. R. (2020). Neonatal Candidemia: Clinical Presentation, Laboratory Profile, Risk Factors and Immediate Outcome in a Tertiary Hospital in Kerala-A Case-Control Study. Global Journal of Medical Research, 2020.

Kumar, S., Bansal, A., Chakrabarti, A., \& Singhi, S. (2013). Evaluation of efficacy of probiotics in prevention of Candida colonization in a PICU—a randomized controlled trial. Critical care medicine, $41(2), 565-572$.

Lagunes, L., Rey-Pérez, A., Martín-Gómez, M. T., Vena, A., de Egea, V., Muñoz, P., \& Rello, J. (2017). Association between source control and mortality in 258 patients with intra-abdominal candidiasis: a retrospective multi-centric analysis comparing intensive care versus surgical wards in Spain. European Journal of Clinical Microbiology \& Infectious Diseases, 36(1), 95-104.

Lahkar, V., Saikia, L., Patgiri, S. J., Nath, R., \& Das, P. P. (2017). Estimation of biofilm, proteinase \& phospholipase production of the Candida species isolated from the oropharyngeal samples in HIV-infected patients. The Indian journal of medical research, 145(5), 635.

Lamoth, F.; Lockhart, S. R.; Berkow, E. L., \& Calandra, T. (2018). Changes in the epidemiological landscape of invasive candidiasis. Journal of Antimicrobial Chemotherapy, 73, i4-i13.

Lausch, K. R., Dungu, K. H. S., Callesen, M. T., Schrøder, H., Rosthøj, S., Poulsen, A., \& Schønheyder, H. C.(2019). Pediatric Candidemia Epidemiology and Morbidities: A Nationwide Cohort. The Pediatric infectious disease journal, 38(5), 464-469.

Leão, M. V. P., Tavares, T. A. A., e Silva, C.R. G., dos Santos, S. S. F., Junqueira, J. C., de Oliveira, L. D., \& Jorge, A. O. C.(2018). Lactobacillus rhamnosus intake can prevent the development of candidiasis. Clinical oral investigations, 22(7), 2511-2518.

Leibovitz, E. (2012). Strategies for the prevention of neonatal candidiasis. Pediatrics \& Neonatology, 53(2), 83-89. 
Li, T., Liu, Z., Zhang, X., Chen, X., \& Wang, S. (2019). Local probiotic lactobacillus crispatus and lactobacillus delbrueckii exhibit strong antifungal effects against vulvovaginal candidiasis in a rat model. Frontiers in Microbiology, 10, 1033.

Liu, M., Huang, S., Guo, L., Li, H., Wang, F., Zhang, Q. I., \& Song, G. (2015). Clinical features and risk factors for blood stream infections of Candida in neonates. Experimental and therapeutic medicine, 10(3), 1139-1144.

Lollis, T. R., Bradshaw, W. T., \& Zukowsky, K. (2014). Fungal Prophylaxis in Neonates. Advances in Neonatal Care, 14(1), 17-23.

López-Cortés, L. E., Almirante, B., Cuenca-Estrella, M., Garnacho-Montero, J., Padilla, B., Puig-Asensio, M., \& Mularoni, A. (2016). Empirical and targeted therapy of candidemia with fluconazole versus echinocandins: a propensity score-derived analysis of a population-based, multicentre prospective cohort. Clinical Microbiology and Infection, 22(8), 733-e1.

Mandelblat, M., Frenkel, M., Abbey, D., Ben Ami, R., Berman, J., \& Segal, E. (2017). Phenotypic and genotypic characteristics of Candida albicans isolates from bloodstream and mucosal infections. Mycoses, 60(8), 534-545.

Mantadakis, E., Pana, Z. D., \& Zaoutis, T. (2018). Candidemia in children: Epidemiology, prevention and management. Mycoses, 61(9), 614-622.

Mantadakis, E., \& Tragiannidis, A. (2019). Invasive Fungal Infections in the Pediatric Intensive Care Unit. The Pediatric infectious disease journal, 38(9), e216-e218.

Manzoni, P. (2007). Use of Lactobacillus casei subspecies Rhamnosus GG and gastrointestinal colonization by Candida species in preterm neonates. Journal of pediatric gastroenterology and nutrition, 45, S190-S194.

Marostica, P. J. C., Villetti, M. C., Ferrelli, R. S., \& Barros, E. (2018). Pediatria: consulta rápida. Artmed.

Marranzino, G., Villena, J., Salva, S., \& Alvarez, S. (2012). Stimulation of macrophages by immunobiotic Lactobacillus strains: influence beyond the intestinal tract. Microbiology and immunology, 56(11), 771-781.

Martin, G. S., Mannino, D. M., Eaton, S., \& Moss, M. (2003). The epidemiology of sepsis in the United States from 1979 through 2000. New England Journal of Medicine, 348(16), 1546-1554.

Martin, R. J., Fanaroff, A. A., \& Walsh, M. C.(2016). Fanaroff e Martin Medicina Neonatal e Perinatal: Doenças do Feto e Infantil: Elsevier Brasil.

Matsubara, V. H., Bandara, H. M. H. N., Mayer, M. P., \& Samaranayake, L. P. (2016). Probiotics as antifungals in mucosal candidiasis. Clinical Infectious Diseases, 62(9), 1143-1153.

May, M. E., \& So, T.-Y. (2014). Overview of probiotics use in the pediatric population. Clinical Pediatrics, 53(13), 1231-1238.

McCarthy, M. W., \& Walsh, T. J. (2017). Molecular diagnosis of invasive mycoses of the central nervous system. Expert review of molecular diagnostics, 17(2), 129-139.

Mccarty, T. P. \& Pappas, P. G. (2016). Invasive Candidiasis. Infectious Disease Clinics of North America, 30(1), 103-124. https://www.sciencedirect.com/science/article/abs/pii/S0891552015001142

Menezes, R. d. P (2018). Candidíase invasiva em neonatos críticos: etiologia, patogenia e perfil molecular (Tese de Doutorado). Universidade Federal de Uberlândia, Uberlândia, Brasil.

Montagna, M. T., Lovero, G., Borghi, E., Amato, G., Andreoni, S., Campion, L., \& Morace, G. (2014). Candidemia in intensive care unit: a nationwide prospective observational survey (GISIA-3 study) and review of the European literature from 2000 through 2013. European Review for Medical and Pharmacological Sciences.

Mylonakis, E., Clancy, C.J., Ostrosky-Zeichner, L., Garey, K. W., Alangaden, G. J., Vazquez, J. A., \& Pappas, P. G. (2015). T2 magnetic resonance assay for the rapid diagnosis of candidemia in whole blood: a clinical trial. Clinical Infectious Diseases, 60(6), 892-899.

Netea, M. G., Joosten, L. A., Van Der Meer, J. W., Kullberg, B.-J., \& Van De Veerdonk, F. L. (2015). Immune defence against Candida fungal infections. Nature Reviews Immunology, 15(10), 630-642.

Olowe, O., Makanjuola, O., Olowe, R., \& Adekanle, D. (2014). Prevalence of vulvovaginal candidiasis, trichomoniasis and bacterial vaginosis among pregnant women receiving antenatal care in Southwestern Nigeria. European Journal of Microbiology and Immunology, 4(4), 193-197.

Oncel, M. Y., Arayici, S., Sari, F. N., Simsek, G. K., Yurttutan, S., Erdeve, O., \& Dilmen, U. (2015). Comparison of Lactobacillus reuteri and nystatin prophylaxis on Candida colonization and infection in very low birth weight infants. The Journal of Maternal-Fetal \& Neonatal Medicine, 28(15), 1790-1794.

Orasch, C., Mertz, D., Garbino, J., Van Delden, C., Emonet, S., Schrenzel, J., \& Imhof, A. (2018). Fluconazole non-susceptible breakthrough candidemia after prolonged low-dose prophylaxis: a prospective FUNGINOS study. Journal of Infection, 76(5), 489-495.

Organization, W. H. (2002). Guidelines for the Evaluation of Probiotics in Food. FAO/WHO Working Group Meeting, 1-11.

Orsi, C.F., Sabia, C., Ardizzoni, A., Colombari, B., Neglia, R. G., Peppoloni, S., \& Blasi, E. (2014). Inhibitory effects of different lactobacilli on Candida albicans hyphal formation and biofilm development. Journal of biological regulators and homeostatic agents, 28(4), 743.

Paho. Pan American Health Organization. (2016). World Health Organization. Epidemiological alert: C.auris outbreak in health care services. 3 October, Washington DC.PAHO/WHO, 1-4.

Pana, Z. D., Roilides, E., Warris, A., Groll, A. H., \& Zaoutis, T. (2017). Epidemiology of invasive fungal disease in children. Journal of the Pediatric Infectious Diseases Society, 6, S3-S11. 
Pandey, N., Gupta, M. K., \& Tilak, R. (2018). Extracellular hydrolytic enzyme activities of the different Candida spp. isolated from the blood of the Intensive Care Unit-admitted patients. Journal of laboratory physicians, 10(4), 392.

Pansieri, C., Pandolfini, C., Jacqz-Aigrain, E., Van Den Anker, J., \& Bonati, M. (2015). Fluconazole prophylaxis in neonates: BMJ Publishing Group Ltd. Archives Disease Childhood, 100(1).

Papadimitriou-Olivgeris, M., Spiliopoulou, A., Kolonitsiou, F., Bartzavali, C., Lambropoulou, A., Xaplanteri, P., \& Christofidou, M. (2019). Increasing incidence of Candidaemia and shifting epidemiology in favor of Candida non-albicans in a 9-year period (2009-2017) in a university Greek hospital. Infection, 47(2), 209-216.

Pappas, P. G., Kauffman, C. A., Andes, D. R., Clancy, C. J., Marr, K. A., Ostroskyzeichner, L. \& Sobel, J. D. (2016). Clinical Practice Guideline for the Management of Candidiasis: 2016 Update by the Infectious Diseases Society of America. Clinical Infectious Diseases, 62(4), e1-e50.

Payen, D. M., Guilhot, J., Launey, Y., Lukaszewicz, A. C., Kaaki, M., Veber, B., \& Robert, R. (2015). Early use of polymyxin B hemoperfu sion in patients with septic shock due to peritonitis: a multicenter randomized control trial. Intensive care medicine, 41(6), 975-984.

Pfaller, M., Neofytos, D., Diekema, D., Azie, N., Meier-Kriesche, H.-U., Quan, S.-P., \& Horn, D. (2012). Epidemiology and outcomes of candidemia in 3648 patients: data from the Prospective Antifungal Therapy (PATH Alliance () registry, 2004-2008. Diagnostic microbiology and infectious disease, 74(4), 323331.

Pharkjaksu, S., Chongtrakool, P., Suwannakarn, K., \& Ngamskulrungroj, P. (2018). Species distribution, virulence factors, and antifungal susceptibility among Candida parapsilosis complex isolates from clinical specimens at Siriraj Hospital, Thailand, from 2011 to 2015. Medical mycology, 56(4), 426-433.

Piqueras, A., Ganapathi, L., Carpenter, J. F., Rubio, T., Sandora, T. J., Flett, K. B., \& Köhler, J. R. (2021). Trends in Pediatric Candidemia: Epidemiology, Anti-Fungal Susceptibility, and Patient Characteristics in a Children's Hospital. Journal of Fungi, 7(2), 78.

Prigent, G., Aït-Ammar, N., Levesque, E., Fekkar, A., Costa, J. M., El Anbassi, S., \& Botterel, F. (2017). Echinocandin resistance in Candida species isolates from liver transplant recipients. Antimicrobial agents and chemotherapy, 61(2).

Qin, Y., Zhang, L., Xu, Z., Zhang, J., Jiang, Y.-y., Cao, Y., \& Yan, T. (2016). Innate immune cell response upon Candida albicans infection. Virulence, 7(5), $512-526$.

Rao, S. C., Athalye-Jape, G. K., Deshpande, G. C., Simmer, K. N., \& Patole, S. K. (2016). Probiotic supplementation and late-onset sepsis in preterm infants: a meta-analysis. Pediatrics, 137(3).

Richardson, J. P., \& Moyes, D. L. (2015). Adaptive immune responses to Candida albicans infection. Virulence, 6(4), 327-337.

Rocha, M. A. R., de Carvaho, C.G., \& Magalhães, S. R. (2019). A importância da higienização oral em recém-nascidos mantidos em Centro de Terapia Intensiva (CTI) neonatal: uma abordagem sobre candidíase invasiva. Revista Uniabeu, 12(32).

Roy, U., Jessani, L. G., Rudramurthy, S. M., Gopalakrishnan, R., Dutta, S., Chakravarty, C., \& Chakrabarti, A. (2017). Seven cases of Saccharomyces fungaemia related to use of probiotics. Mycoses, 60(6), 375-380.

Sabia, C., Anacarso, I., Bergonzini, A., Gargiulo, R., Sarti, M., Cond, C., \& Bondi, M. (2014). Detection and partial characterization of a bacteriocin-like substance produced by Lactobacillus fermentum CS57 isolated from human vaginal secretions. Anaerobe, 26, 41-45.

Salvatore, C.M., Chen, T. K., Toussi, S. S., DeLaMora, P., Petraitiene, R., Finkelman, M. A., \& Walsh, T. J. (2016). (1 $\rightarrow$ 3)- $\beta$-d-Glucan in cerebrospinal fluid as a biomarker for Candida and Aspergillus infections of the central nervous system in pediatric patients. Journal of the Pediatric Infectious Diseases Society, 5(3), 277-286.

Santolaya, M. E., Matute, T. A., de Queiroz Telles, F., Colombo, A. L., Zurita, J., Tiraboschi, I. N., \& Sifuentes, J. (2013). Recomendaciones para el manejo de la candidemia en neonatos en América Latina. Revista iberoamericana de micologia, 30(3), 158-170.

Sasso, M., Roger, C., Sasso, M., Poujol, H., Barbar, S., Lefrant, J. Y., \& Lachaud, L. (2017). Changes in the distribution of colonising and infecting Candida spp. isolates, antifungal drug consumption and susceptibility in a French intensive care unit: A 10-year study. Mycoses, 60(12), 770-780.

Saxena, S., Gee, J., Klieger, S., Kajon, A., Petersen, H., Zaoutis, T., \& Fisher, B. (2018). Invasive fungal disease in pediatric solid organ transplant recipients. Journal of the Pediatric Infectious Diseases Society, 7(3), 219-225.

Seneviratne, C.J., Rajan, S., Wong, S. S., Tsang, D. N., Lai, C. K., Samaranayake, L. P., \& Jin, L. (2016). Antifungal susceptibility in serum and virulence determinants of Candida bloodstream isolates from Hong Kong. Frontiers in Microbiology, 7, 216.

Sierra, C.E. (2009). Candidemias Nosocomiais: Padrões de alteração clínico-epidemiológica, fatores prognósticos e influência do tratamento antifúngico precoce na sua evolução. Estudo 2002-2005( Tese de Doutorado), Universidade de Murcia, Murcia, Espanha.

Sopian, I. L., Sa'adiah Shahabudin, M. A. A., Lung, L. T. T., \& Sandai, D. (2016). Yeast infection and diabetes mellitus among pregnant mother in Malaysia. The Malaysian journal of medical sciences: MJMS, 23(1), 27.

Sousa, R. A. (2019). Fatores de risco para candidemia em neonatos em um hospital universitário de Belo Horizonte (Dissertação de Mestrado). Universidade Federal de Minas Gerais, Minas Gerais, Brasil.

Spurbeck, R. R., \& Arvidson, C.G. (2010). Lactobacillus jensenii surface-associated proteins inhibit Neisseria gonorrhoeae adherence to epithelial cells. Infection and immunity, 78(7), 3103-3111.

Szajewska, H. (2016). What are the indications for using probiotics in children? Archives of disease in childhood, 101(4), 398-403. 
Research, Society and Development, v. 10, n. 4, e53710414326, 2021

(CC BY 4.0) | ISSN 2525-3409 | DOI: http://dx.doi.org/10.33448/rsd-v10i4.14326

Teo, J. Q.-M., Candra, S. R., Lee, S. J.-Y., Chia, S. Y.-H., Leck, H., Tan, A.-L., \& Ee, R. P.-L. (2017). Candidemia in a major regional tertiary referral hospital-epidemiology, practice patterns and outcomes. Antimicrobial Resistance \& Infection Control, 6(1), 1-11.

Toth, R., Toth, A., Vagvolgyi, C., \& Gacser, A. (2017). Candida parapsilosis secretou lipase como um importante fator de virulência. Current Protein and Peptide Science, 18 (10), 1043-1049.

Tragiannidis, A., Fegeler, W., Rellensmann, G., Debus, V., Müller, V., Hoernig-Franz, I., \& Groll, A. (2012). Candidaemia in a European Paediatric University Hospital: a 10-year observational study. Clinical Microbiology and Infection, 18(2), E27-E30.

Vallabhaneni, S., Cleveland, A. A., Farley, M. M., Harrison, L. H., Schaffner, W., Beldavs, Z. G., \& Smith, R. M. (2015, December). Epidemiology and risk factors for echinocandin nonsusceptible Candida glabrata bloodstream infections: data from a large multisite population-based candidemia surveillance program, 2008-2014. Open forum infectious diseases, 2(4).

Vasileiou, E., Apsemidou, A., Vyzantiadis, T.-A., \& Tragiannidis, A. (2018). Invasive candidiasis and candidemia in pediatric and neonatal patients: A review of current guidelines. Current medical mycology, 4(3), 28.

Walsh, T. J., Katragkou, A., Chen, T., Salvatore, C. M., \& Roilides, E. (2019). Invasive candidiasis in infants and children: recent advances in epidemiology, diagnosis, and treatment. Journal of Fungi, 5(1), 11.

Wang, G., Dai, C., Liu, Y., \& Li, Y. (2013). Cerebral and renal abscess and retino-choroiditis secondary to Candida albicans in preterm infants: eight case retrospective study. Clin Exp Obstet Gynecol, 40(4), 519-523.

Wang, X., van de Veerdonk, F. L., \& Netea, M. G. (2016). Basic genetics and immunology of Candida infections. Infectious Disease Clinics, 30(1), 85-102.

Zeng, Z., Tian, G., Ding, Y., Yang, K., Deng, J., \& Liu, J. (2020). Epidemiology, antifungal susceptibility, risk factors and mortality of invasive candidiasis in neonates and children in a tertiary teaching hospital in Southwest China. Mycoses, 63(11), 1164-1174.

Zhang, G.-Q., Hu, H.-J., Liu, C.-Y., Shakya, S., \& Li, Z.-Y. (2016). Probiotics for preventing late-onset sepsis in preterm neonates: a PRISMA-compliant systematic review and meta-analysis of randomized controlled trials. Medicine, 95(8).

Zhang, L., Yu, S. Y., Chen, S. C.A., Xiao, M., Kong, F., Wang, H., \& Xu, Y. C.(2020). Molecular characterization of Candida parapsilosis by microsatellite typing and emergence of clonal antifungal drug resistant strains in a multicenter surveillance in China. Frontiers in Microbiology, 11, 1320.

Zuza-Alves, D. L., Silva-Rocha, W. P., \& Chaves, G. M. (2017). An update on Candida tropicalis based on basic and clinical approaches. Frontiers in microbiology, 8, 1927. 